\title{
ÉCOLOGIE DES OCTOCORALLIAIRES COMMUNS DU SUBSTRAT DUR DANS LA RÉGION DE BANYULS-SUR-MER \\ Essai d'une méthode
}

\author{
par \\ STEVEN WEINBERG \\ Institut de Zoologie taxonomique, Université d'Amsterdam, Pays-Bas
}

\begin{abstract}
RESUME
Un travail d'écologie a été entrepris, suivant une méthodologie nouvelle, sur le benthos rocheux de la région de Banyuls-sur-Mer (Pyrénées-Orientales, France). Les mesures et le calcul ont permis de mettre en évidence les rapports existants entre certains paramètres physiques et la répartition des animaux étudiés: les Octocoralliaires. La lumière s'est avérée être le facteur écologique le plus important dans les stations sous-marines étudiées.
\end{abstract}

\section{ABSTRACT}

An underwater ecological study, using a new methodological approach, was carried out on the hard substratum near Banyuls-sur-Mer (Pyrénées-Orientales, France). The data obtained were analyzed with a computer program, which showed the existing relationships between some physical parameters and the distribution of the animals studied: the Octocorallia. Light proved to be the most important ecological factor in the underwater stations studied.

\section{INTRODUCTION}

L'écologie sous-marine est une science naissante. Alors que nos connaissances sur la faune et la flore sous-marine n'ont cessé de croître, surtout depuis que le scaphandre autonome est devenu un appareil d'utilisation courante, nos connaissances sur le micro-climat sous-marin n'ont guère évolué.

Or ce sont justement l'existence de variations brusques des conditions physiques d'un endroit à l'autre, jointes aux préférences écologiques souvent mal connues des organismes sous-marins, qui expliquent l'absence de monotonie rencontrée dans les biotopes accessibles au plongeur autonome.

Plusieurs auteurs ont indiqué brièvement la façon dont les facteurs agissent sur les organismes (Théodor, 1963; Théodor \& Denizot, 1965; Laubier, 1966; Riedl, 1966; Roos, 1967; Barletta et al., 1968; Chapman \& Théodor, 1969; Chardy,
1970; Schuhmacher, 1973; Velimirov, 1973). On parle alors d'animaux plus au moins sciaphiles, ou d'animaux préférant des eaux calmes, ou au contraire, battues.

D'autres auteurs se sont efforcés de mesurer de façon précise les fluctuations des facteurs du milieu (Landais, 1955; Forstner \& Rützler, 1970; Jaubert, 1972), quoique des mesures continues (pendant une ou plusieurs années) soient pratiquement inexistantes. Il est encore plus rare de trouver la combinaison d'observations sur le terrain jointes à des mesures précises et continues.

Ceci est probablement dû à deux causes distinctes, la première étant le manque d'intégration qui existe entre le travail des océanographes biologistes et physiciens, la seconde, la difficulté que rencontre le plongeur à recueillir assez de données précises dans le peu de temps qui lui est accordé pendant chaque plongée.

C'est justement une méthode de travail adaptée à la plongée en scaphandre autonome que nous nous sommes proposé d'élaborer, ce travail comportant aussi bien des observations sur les organismes que sur les facteurs du milieu.

Dans la région de Banyuls-sur-Mer (PyrénéesOrientales, France) nous avons choisi des stations sous-marines, toutes situées sur le substrat dur, où nous avons fait l'inventaire des Octocoralliaires (Coelentérés, Anthozoaires). En même temps nous avons mesuré quelques paramètres physiques. Ces mesures ont été effectuées à quatre reprises, réparties sur une année, ceci afin de rendre compte également des variations saisonnières.

Pour effectuer ce travail, environ 120 plongées ont été faites à l'aide du scaphandre autonome. Le travail de prospection (choix des stations) a été effectué pendant le mois d'août 1972. Les périodes de recherche proprement dite se situent en aoûtseptembre et novembre 1973, et février et juinjuillet 1974. 


\section{LA LUMIERE}

\section{Les facteurs qui agissent sur la lumière}

Avant d'atteindre une station sous-marine, la lumière subit plusieurs atténuations successives dues à des causes diverses. La première est la réflexion au niveau de l'interface air-eau. Cette réflexion, elle-même, dépend de plusieurs paramètres, tel l'état nuageux du ciel, l'état de la mer et l'élévation du soleil au-dessus de l'horizon. Les données sur ce phénomène étant assez rares, nous lui avons consacré quelques mesures.

La lumière non-réfléchie, c'est à dire la lumière existant à $0 \mathrm{~m}$ de profondeur ( $0^{-}$, lire: zéro-moins) va ensuite subir une atténuation suivant la loi de Lambert-Beer:

$$
I_{p}=I_{o^{-}} \cdot e^{-k p}
$$

avec

$I_{p}=$ intensité lumineuse à $p$ mètres de profondeur,

$I_{0^{-}}=$intensité lumineuse juste sous la surface,

$k=$ coefficient d'extinction de l'eau de mer.

La constante $k$ dépend de la longueur d'onde, la loi de Lambert-Beer n'étant valable que pour des radiations monochromatiques. Ce sont ces différentes valeurs de $k$ qui sont responsables de la distribution spectrale sous-marine, l'eau de mer agissant comme un monochromateur qui élimine rapidement certaines radiations (surtout le rouge et l'infra-rouge). En Méditerranée, pour les profondeurs comprises entre 10 et $40 \mathrm{~m}$, la courbe de distribution spectrale présente un maximum vers $480 \mathrm{~nm}$, avec une largeur de bande de $100 \mathrm{~nm}$ environ (Jerlov, 1968). Dans le domaine de longueurs d'onde où nous avons mesuré (et qui correspond approximativement à la courbe de distribution précitée), nous pouvons supposer $k$ constant sans introduire une erreur appréciable.

En revanche, $k$ varie tout au long de l'année (et même présente des variations diurnes) avec la turbidité de l'eau, dont il est justement l'indicateur. Moins l'eau est claire, plus $k$ est grand. La profondeur de disparition du disque de Secchi, qui est l'indicateur le plus utilisé en océanographie pour mesurer la turbidité de l'eau de mer, peut être reliée à $k$ par la formule $k=q / P_{s d}$ où $q$ est une constante et $\boldsymbol{P}_{\boldsymbol{s}}$ la profondeur de disparition du disque de Secchi (Atkins \& Poole, 1930). Les profondeurs moyennes de disparition du disque sont connues pour la région de Banyuls (Bhaud et al., 1967). Des données supplémentaires pour les années 1971-1973 nous ont été fournies par Monsieur M. Panouse du Laboratoire Arago. En revanche, les valeurs de $q$ varient suivant l'auteur
(Holmes, 1957) et nous avons, au cours de nos mesures, corrélé nos résultats avec la profondeur de disparition du disque de Secchi, pour aboutir ainsi à la valeur de $q$ pour les eaux côtières de Banyuls, et pour la gamme de longueurs d'onde considérée. Ainsi, à partir des $\boldsymbol{P}_{\boldsymbol{s} a}$ connues, nous avons pu calculer les $k$ moyens pour chaque mois (cf. fig. 2).

Les considérations précédentes permettent d'expliquer la diminution de la luminosité avec la profondeur. Pour les organismes sessiles marins, cependant, un autre facteur est également très important, c'est le degré d'exposition de leur substrat à la lumière. Ainsi, à la même profondeur, une surface horizontale, un plan vertical, une grotte, ne recevront pas la même quantité de lumière. Il est donc nécessaire de qualifier chaque station par une constante, comprise entre 0 et 1 , et rendant compte de son degré d'exposition.

\section{Les mesures}

Pour nos mesures nous nous sommes servi d'un appareil (Weinberg, 1974) permettant de comparer l'énergie lumineuse reçue à une station sous-marine donnée (cette station pouvant se trouver en pleine eau) avec celle reçue en surface. L'appareil comprend deux cellules photo-électriques au Sélénium, l'une étanche, dite „cellule sous-marine”, l'autre étant la "cellule de surface". Chaque cellule, dotée d'un filtre bleu-vert, possède une courbe de sensibilité relative correspondant grossièrement à la distribution spectrale rencontrée sous l'eau. Avec un maximum à $480 \mathrm{~nm}$ et une largeur de bande de $60 \mathrm{~nm}$, la bande passante de l'appareil est plus étroite que celle de l'eau de mer, ce qui réduit les erreurs qui découlent de mesures effectuées à diverses profondeurs. Chaque cellule est équipée d'un collecteur hémisphérique en matière plastique dépolie. Une fois plongée dans l'eau, la demi-sphère délimitée par le collecteur se comporte comme une lentille divergente (l'indice de réfraction à l'intérieur étant moins élevé qu'en dehors). Il convient donc d'apporter une correction à chaque mesure. Nous avons établi expérimentalement la valeur de la constante de correction.

Les deux cellules sont reliées à un appareil restant sur le bateau de surveillance, où un observateur de surface lit directement le pourcentage de lumière que reçoit la cellule sous-marine par rapport à celle que reçoit la cellule de surface. Un plongeur descend avec la cellule sous-marine, et effectue des mesures à diverses profondeurs et à diverses stations. Pour chaque mesure, il avertit 
l'observateur de surface au moyen d'un signal sonore. A partir des mesures ainsi obtenues, il est possible de tracer une courbe logarithmique dont on tire plusieurs renseignements: la réflexion $\boldsymbol{R}$ à la surface') (que l'on peut corréler à la hauteur du soleil, l'état nuageux du ciel et l'état de la mer), le coefficient d'extinction $k$ (que l'on peut corréler à la profondeur de disparition du disque de Secchi, $\left.P_{s d}\right)$ et le coefficient d'exposition $S$ de la station considérée. voyons deux $\boldsymbol{P}_{10}$ différents, donnant lieu à deux $k$ différents $(0,067$ et 0,184$)$. Ceci correspond à une stratification: près du fond il y a une couche plus turbide.

Enfin, la courbe 3 comporte également les points $\mathrm{A}$ et $\mathrm{B}$, correspondant à des stations. A est une grotte, $B$ une surface verticale, ces stations recevant respectivement $0,1 \%$ et $1,25 \%$ par rapport à la surface, alors que la lumière reçue à la profondeur des stations $\mathrm{A}$ et $\mathrm{B}(36 \mathrm{~m})$ est de $2,6 \%$. Le

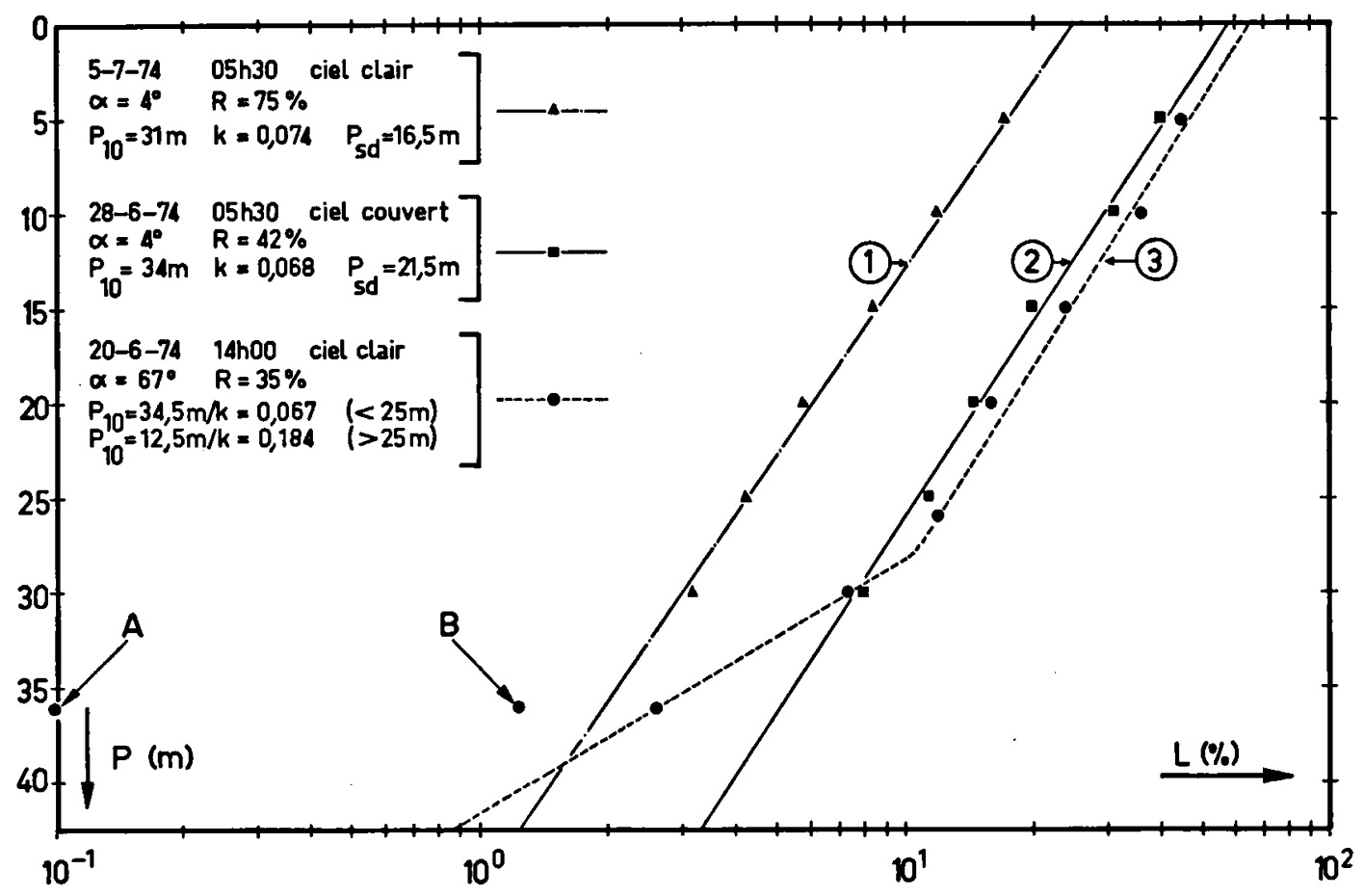

Fig. 1. Trois exemples de coupes optiques effectuées au large du Cap de l'Abeille ( $L=$ lumière, $P=$ profondeur).

La fig. 1 donne quelques exemples de ces mesures. La réflexion en surface est de $75 \%, 42 \%$ et $35 \%$ respectivement pour les trois courbes. Les courbes 1 et 2 correspondent toutes les deux à une mer calme et à une élévation solaire de $4^{\circ}$. La courbe 1 correspond à un ciel clair, la courbe 2 à un ciel couvert. Par ciel clair la réflexion diminue avec des hauteurs de soleil croissantes, comme en témoignent les courbes 1 et 3 (voir aussi fig. 3).

On calcule $k$ d'après la formule suivante: $k=$ $2,3 / P_{10}$ où $P_{10}$ est la hauteur de la colonne d'eau sur laquelle la luminosité diminue jusqu'à $10 \%$ de sa valeur initiale. Les courbes 1 et 2 donnent des $k$ de 0,074 et de 0,068 . Pour la courbe 3 , nous

1) $R$ représente ici la réflexion totale (parfois appelée albédo) qui est la réflexion proprement dite (à l'interface air-eau) + la lumière rétrodiffusée par la colonne d'eau considérée. coefficient d'exposition $S$ des stations A et B sera donc de 0,04 et de 0,48 respectivement.

De ces mesures nous avons pu déduire que la constante $q$ (voir paragraphe précédent) pour l'eau côtière de Banyuls et pour des longueurs d'onde de $480 \mathrm{~nm}$ environ est de 1,39 en moyenne. Avec les $\boldsymbol{P}_{s d}$ connues (voir références plus haut) nous avons obtenu la courbe de variation de $k$ au long de l'année qui est représentée par la fig. 2. L'effet des fluctuations saisonnières n'est donc pas négligeable, contrairement à ce qu'en dit Jaubert (1972). Non seulement les variations de $k$ se font nettement sentir, mais il vient s'y ajouter les différences de réflexion en surface au cours de l'année.

Quant aux valeurs de $R$, elles sont moins fiables, parce que le nombre de mesures effectuées est trop faible. A titre d'indication nous donnons la fig. 3 , où le pourcentage de réflexion 


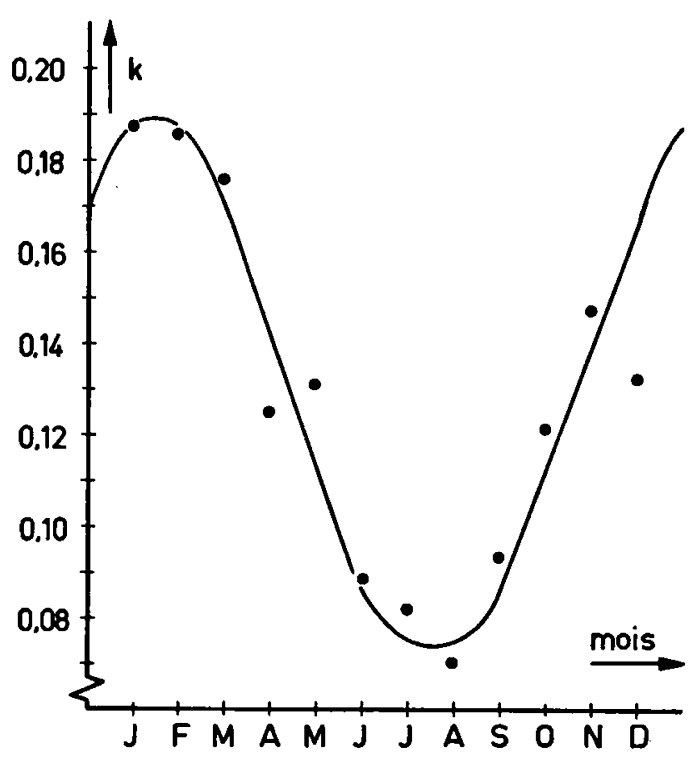

Fig. 2. Variations annuelles du coefficient d'extinction $k$ dans la région de Banyuls-sur-Mer.

est fourni en fonction de l'élévation solaire $\alpha$, pour des jours ensoleillés. Une limite supérieure est donnée pour mer agitée et une limite inférieure pour mer calme. Pour nos calculs (paragraphe suivant) nous nous sommes servi de la courbe moyenne. Par temps couvert, le coefficient de réflexion reste pratiquement constant, avec une valeur moyenne de $48 \%$.

Jusqu'ici nous n'avons présenté que les mesures permettant un calcul relatif, c'est à dire permettant de calculer le pourcentage de la lumière arrivant en surface qui atteindra la station considérée. Afin de transformer ces données en données énergétiques absolues, il est nécessaire de connaître l'intensité de l'énergie lumineuse à la surface à chaque moment de l'année. Nous sommes heureux que Monsieur H. Boutière du Laboratoire Arago ait

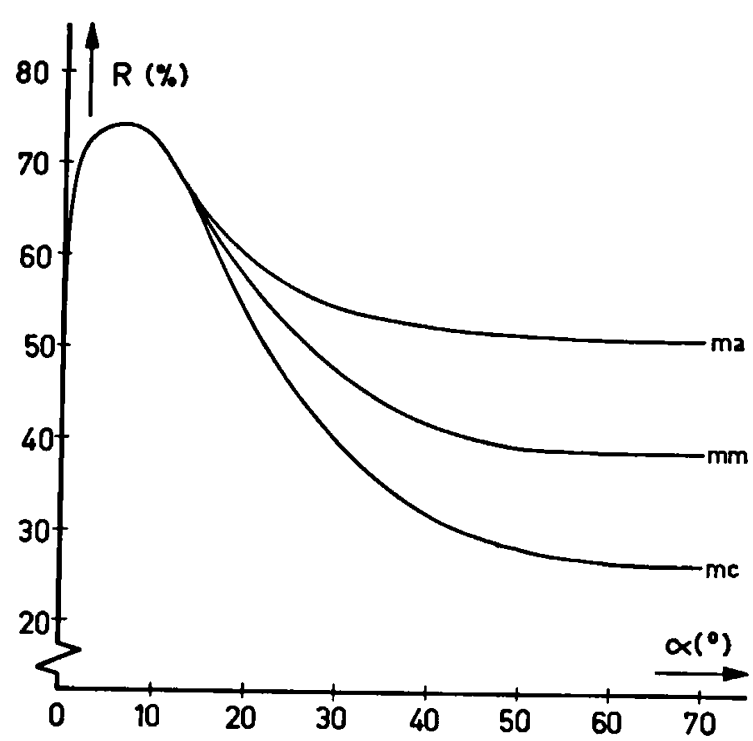

Fig. 3. Coefficient de réflexion $\boldsymbol{R}$ pour temps ensoleillé en fonction de l'élévation solaire $\alpha$ et de l'état de la mer: $\mathrm{ma}=$ mer agitée, $\mathrm{mm}=$ mer moyenne, $\mathrm{mc}=$ mer calme.

bien voulu mettre à notre disposition des enregistrements qu'il a effectué à l'aide d'un solarimètre pendant les années 1966-1968. Nous avons dépouillé ces enregistrements, et nous avons classé l'énergie lumineuse par jour moyen du mois et en 8 périodes de 2 heures chacune. Ces données sont présentées dans le tableau I.

Avant de se servir des données ainsi obtenues, il convient de se rappeler que nous n'avons effectué nos mesures relatives que dans une bande spectrale assez étroite. Le solarimètre, qui est un appareil à thermopile, possède une réponse spectrale "plate" de 300 à $3000 \mathrm{~nm}$ environ. La conséquence de ceci est que ces mesures représentent tout le spectre énergétique tel qu'il est reçu à la surface terrestre.

Tableau I. Insolation en cal/ $\mathrm{cm}^{2} /$ heure (moyennes 1966-1968) à Banyuls-sur-Mer.

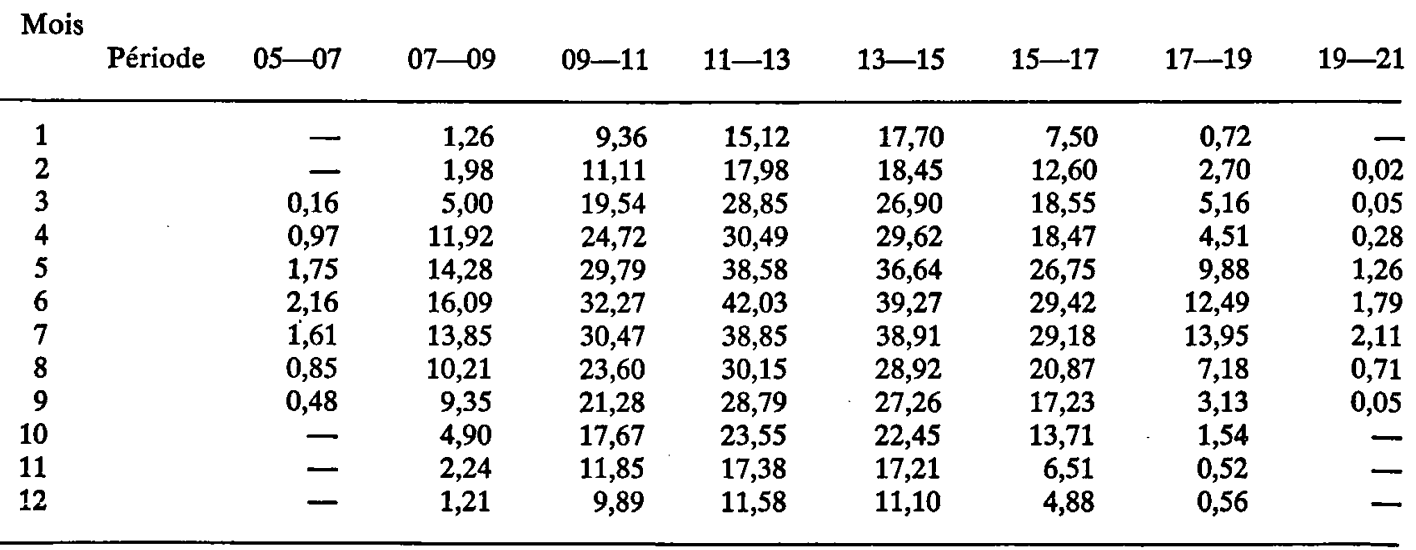




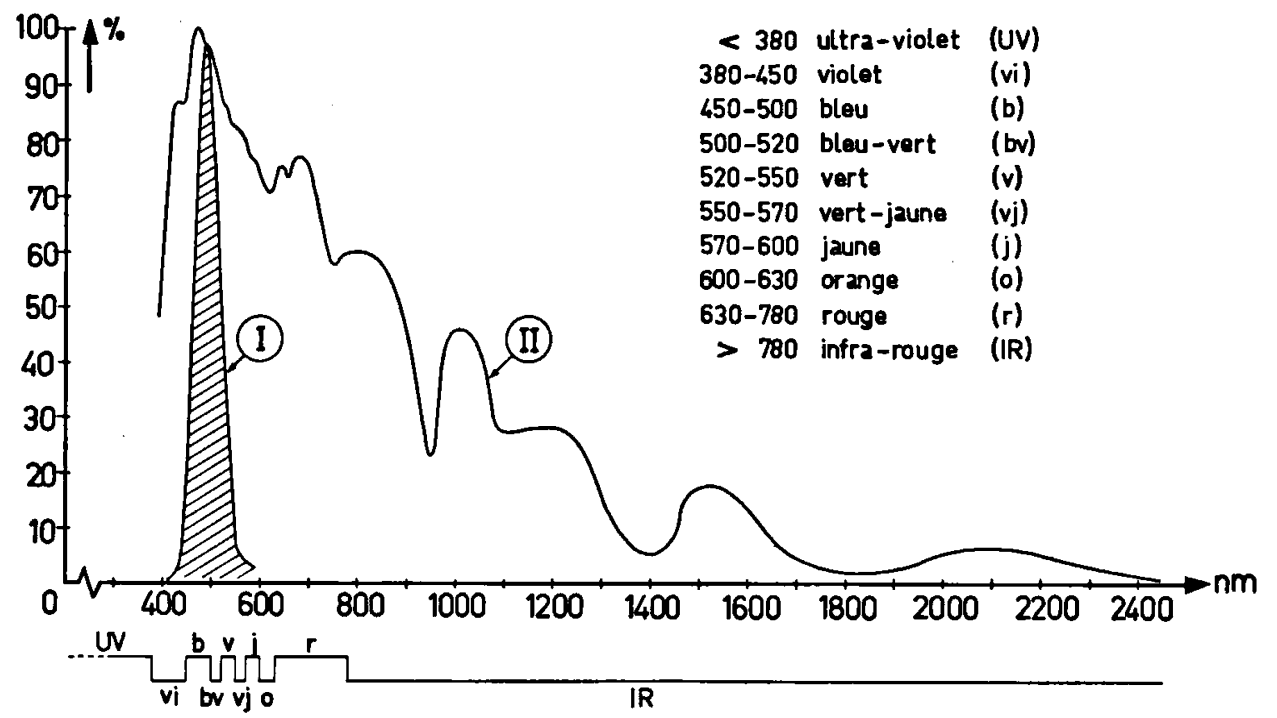

Fig. 4. Comparaison de la distribution spectrale de l'énergie lumineuse que nous avons mesurée (courbe I) avec celle de l'énergie totale reçue à la surface terrestre (courbe II, d'après Jerlov, 1968).

Ces deux courbes sont portées sur la fig. 4. La surface sous chacune des courbes représente l'énergie totale définie par chacune des distributions spectrales considérées. Le rapport de l'énergie lumineuse telle que nous l'avons mesurée sous l'eau avec celle qui arrive en surface est de $11,2 \%$, ceci étant dû surtout à la disparition de l'infrarouge, du rouge et de l'orange. Les mesures absolues doivent donc être multipliées par une constante, $K=0,112$, avant de subir le calcul aboutissant à l'énergie reçue par chacune des stations.

\section{Le calcul}

Si nous reprenons tous les facteurs présentés dans les deux paragraphes précédents, nous arrivons au schéma de la fig. 5 .

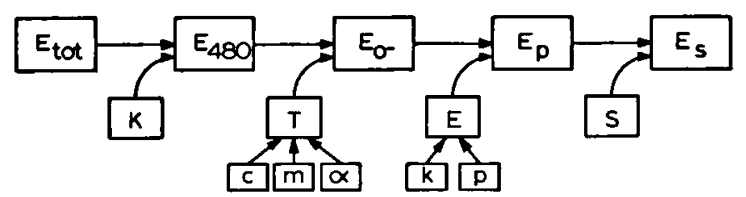

Fig. 5. Schéma des facteurs qui influent sur l'énergie lumineuse.

$E_{t o t}=$ énergie totale mesurée à l'aide du solarimètre,

$E_{\mathbf{4 8 0}}=$ énergie dans la bande spectrale de maximum $480 \mathrm{~nm}$, de largeur $60 \mathrm{~nm}$,

$E_{0^{-}}=$énergie juste sous la surface de l'eau,

$E_{p}=$ énergie à la profondeur $p$,
$E_{8}=$ énergie à la station considérée,

$K=$ constante de conversion $(0,112)$,

$T=$ coefficient de transmission $(T=1-R, R$ étant la réflexion),

$E=$ extinction $\left(E=e^{-k p}\right)$,

$S=$ coefficient d'exposition de la station considérée,

$c=$ état du ciel,

$m$ = état de la mer,

$\alpha=$ élévation solaire,

$k=$ coefficient d'extinction de l'eau de mer,

$p=$ profondeur de la station considérée.

Beaucoup de ces grandeurs étant des variables, nous avons fait une sommation des mois, afin d'arriver au bilan annuel. Pour cela, nous avons pris le $k$ moyen de chaque mois (voir fig. 2). Nous avons également pris un ,jour-type" de chaque mois. Ce jour est constitué ainsi: la durée moyenne de l'insolation totale de chaque mois est connue (Jacques et al., 1969b). Ce nombre, divisé par le nombre de jours du mois considéré, donne la longueur de l'insolation journalière $L_{8}$ du mois considéré. La longueur astronomique $L_{a}$ du jour moyen du mois (le 15 de chaque mois) diminué de la longueur d'insolation journalière donne la longueur de temps couvert $L_{c}$ par jour:

$$
L_{s}+L_{c}=L_{a}
$$

A partir du $m$ moyen, on calcule le jour-soleil type (le $15 \mathrm{du}$ mois, en supposant $L_{s}=L_{a}$ ) avec les $T$ correspondants aux divers $\alpha$. On calcule également le jour-nuage type (le 15 du mois, en supposant $L_{c}-L_{a}$ ) avec $T=0,52$. Ensuite, on aboutit au jour-type du mois avec la formule: 
$L_{b} / L_{a} \cdot$ jour-soleil type $+L_{c} / L_{a} \cdot$ jour-nuage type $=$ jour-mois type.

L'énergie reçue pendant une année à la station $i$ sera ensuite:

$E_{i}=S_{i} K \sum_{m=1}^{12} d_{m} e^{-k_{m} p_{i}}\left[S_{m} \sum_{h=1}^{26} E_{m h} T_{m h}+\left(1-S_{m}\right) T \cdot \sum_{h=1}^{26} E_{m h}\right]$

avec:

$E_{i}=$ énergie annuelle à la station $i$,

$S_{i}=$ coefficient d'exposition de la station $i$,

$K=$ constante de conversion $(K=0,112)$,

$d_{m}=$ durée du mois $m$ en jours,

$k_{m}=$ coefficient d'extinction moyen du mois $m$,

$p_{i}=$ profondeur de la station $i$,

$s_{m}=$ pourcentage d'heures ensoleillées mois $m$ $\left(s_{m}=L_{s} / L_{a} ; 1-s_{m}=L_{c} / L_{a}\right)$,

$E_{m h}=$ énergie totale reçue en surface, mois $m$, heure $h$,

$T_{m h}=$ transmission temps ensoleillé, mois $m$, heure $h$,

$T^{\prime}=$ transmission temps nuageux $\left(T^{\prime}=0,52\right)$.

Les résultats pour les diverses stations seront présentés au paragraphe VII. Cependant, nous publions ici (tableau II) les résultats pour diverses profondeurs, en pleine eau. On peut constater qu'en ce qui concerne le facteur lumière, les variations saisonnières sont d'autant plus accentuées que l'on descend en profondeur.

Nous tenons à signaler que les valeurs de l'énergie totale en surface diffèrent de celles que donnent Jacques et al. (1969b). Ces auteurs ont calculé $E_{\text {tot }}$ d'après le nombre d'heures d'ensoleillement mensuel à l'aide d'une formule. Il est étrange que ces auteurs, qui ont également effectué des mesures de l'énergie (Jacques et al., 1968), n'aient pas remarqué que le résultat de leur calcul ne corresponde pas à celui de leurs mesures. Or ces dernières sont en accord avec les chiffres que nous donnons ici. Il faut conclure que la formule utilisée par Jacques et al. (1969b) est fausse.

\section{LE COURANT}

Un facteur écologique intéressant est le courant. Plusieurs auteurs (Théodor, 1963; Théodor \& Denizot, 1965; Riedl, 1966; Schuhmacher, 1973; Velimirov, 1973) ont indiqué l'influence que peut avoir le courant (ou le mouvement d'eau principal) à la fois sur la forme et l'orientation des colonies de Gorgones. Nous résumerons ici les observations de ces auteurs.

Les colonies de Gorgones, qui se présentent comme un éventail, se situent dans un plan perpendiculaire au sens de la turbulence principale (Riedl, 1966; Théodor, 1963). Dans le cas de courants très réguliers, les Gorgones se développent de façon parfaitement plane, ou légèrement convexe, la face convexe faisant face au courant (Théodor, 1963). Dans le cas de courants irréguliers, les colonies peuvent développer des branches dans tous les sens. Dans de rares cas, Riedl et Théodor rencontrent des colonies en forme de „fouet". Alors que le premier auteur explique cette forme adaptative par le facteur lumière (les colonies, dans des endroits enfermés, s'orientent vers la lumière), le second auteur l'explique par la turbulence (dans les tourbillons, le fouet résiste le mieux à l'arrachement). Enfin, les expériences de Velimirov (1973) ont bien démontré la sensibilité des Gorgones au sens du courant: des colonies planes, tournées avec leur substrat d'un quart de tour, développaient pendant leur croissance des ramifications perpendiculaires au plan initial. Les observations de Schuhmacher (1973) sur des espè-

Tableau II. Energie solaire en cal/cm²/jour.à Banyuls-sur-Mer.

\begin{tabular}{|c|c|c|c|c|c|c|c|}
\hline Mois & $E_{t, t}$ & $E_{480}$ & $E_{0^{-}}$ & $E_{10^{-}}$ & $E_{20^{-}}$ & $E_{30^{-}}$ & $E_{40^{-}}$ \\
\hline 1 & 103,32 & 11,57 & 5,280 & 0,814 & 0,125 & 0,019 & 0,003 \\
\hline 2 & 129,67 & 14,52 & 7,068 & 1,100 & 0,171 & 0,027 & 0,004 \\
\hline 3 & 208,41 & 23,34 & 12,038 & 2,156 & 0,386 & 0,069 & 0,012 \\
\hline 4 & 241,96 & 27,10 & 14,511 & 3,651 & 0,918 & 0,231 & 0,058 \\
\hline 5 & 317,87 & 35,60 & 19,321 & 6,304 & 2,057 & 0,671 & 0,219 \\
\hline 6 & 351,04 & 39,32 & 21,453 & 8,988 & 3,765 & 1,578 & 0,661 \\
\hline 7 & 337,85 & 37,84 & 20,558 & 9,614 & 4,496 & 2,103 & 0,983 \\
\hline 8 & 244,99 & 27,44 & 14,795 & 7,059 & 3,368 & 1,607 & 0,767 \\
\hline 9 & 215,14 & 24,10 & 12,514 & 5,191 & 2,153 & 0,893 & 0,370 \\
\hline 10 & 167,64 & 18,78 & 9,305 & 3,160 & 1,073 & 0,364 & 0,124 \\
\hline 11 & 111,44 & 12,48 & 5,856 & 1,518 & 0,394 & 0,102 & 0,026 \\
\hline 12 & 78,45 & 8,79 & 3,878 & 0,737 & 0,140 & 0,027 & 0,005 \\
\hline
\end{tabular}


ces différentes du genre Acabaria en Mer Rouge confirment ce qui précède: $A$. pulchra, qui vit dans la zone des brisants, développe des ramifications dans tous les sens, avec les polypes disposés tout autour des branches; $A$. biserialis, en revanche, qui vit dans les zones plus profondes, à courants réguliers, développe des ramifications dans un seul plan, les polypes étant disposés de part et d'autre des branches, dans le plan de la colonie.

Nous avons voulu tester quantitativement l'influence du courant, c'est à dire voir si certaines espèces ont des préférences pour certaines vitesses du courant.

Pour la mesure du courant nous nous sommes initialement heurté à des problèmes d'ordre technique: le premier courantomètre que nous avons construit était du type „anémomètre”, le second était à hélice. Ni le premier (qui avait l'avantage d'être indépendant du sens du courant), ni le second, n'étaient assez sensibles pour enregistrer les courants faibles rencontrés au fond (de l'ordre de $150 \mathrm{~m} / \mathrm{h}$ en moyenne). Nous avons alors construit le "courantomètre à nuage d'encre" représenté par la fig. 6. Il s'agit d'un tube à gros diamètre en plexiglas, muni d'une poignée. Fixée sur la poignée, on trouve une seringue reliée par un fin tuyau à un aileron se trouvant à l'entrée du tube. On remplit la seringue d'encre. Au fond, le plongeur tient le courantomètre de façon que l'axe du tube corresponde à la direction du courant (il y a toujours des particules en suspension dans l'eau de mer, qui indiquent le sens du courant). Une faible pression sur la seringue fait jaillir un nuage d'encre à l'entrée du tube, juste au centre. Ce nuage est porté par le courant dominant. Le temps (en secondes) que met le nuage à parcourir la distance connue entre deux anneaux peints sur le tube donne la vitesse du courant. Ce système a l'avantage de devenir de plus en plus précis avec des courants de plus en plus faibles: le nombre de secondes augmente, et l'erreur relative sur le temps va en diminuant.

Nous avons effectué ainsi plusieurs mesures (de 2 à 9) à chaque station. Ce faible nombre d'obser-

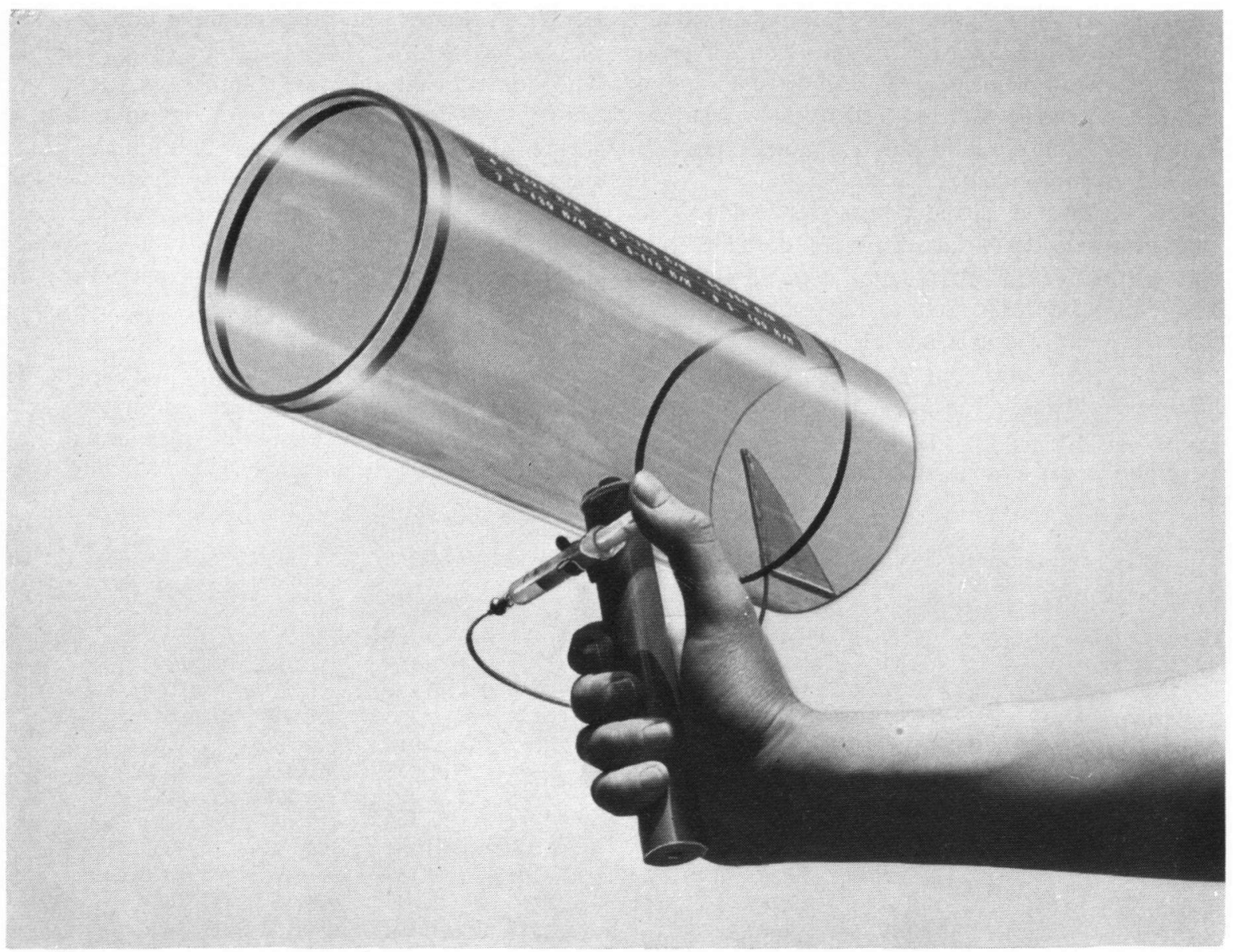

Fig. 6. Le courantomètre à nuage d'encre que nous avons employé. 
vations permet de comparer les stations, mais les valeurs absolues ne sont données qu'à titre indicatif: des mesures plus suivies seraient souhaitables. Ces résultats seront présentés au paragraphe VII.

\section{LA TEMPÉRATURE}

\section{Le cycle annuel}

Le cycle annuel de la température de l'eau de mer a été étudié depuis longtemps par les collaborateurs du Laboratoire Arago de Banyuls-sur-Mer. Plusieurs publications lui sont (en partie) consacrées (Thiriot, 1966; Bhaud et al., 1967; Jacques et al., 1968, 1969a, 1969b, 1971).

Dans chacune de ces publications figure une liste de mesures effectuées tout au long de l'année, à plusieurs profondeurs. Nous avons calculé, d'après ces données, les moyennes de la température pour chaque mois et chaque profondeur. A partir de ces chiffres nous avons dressé le graphique de la fig. 7 .

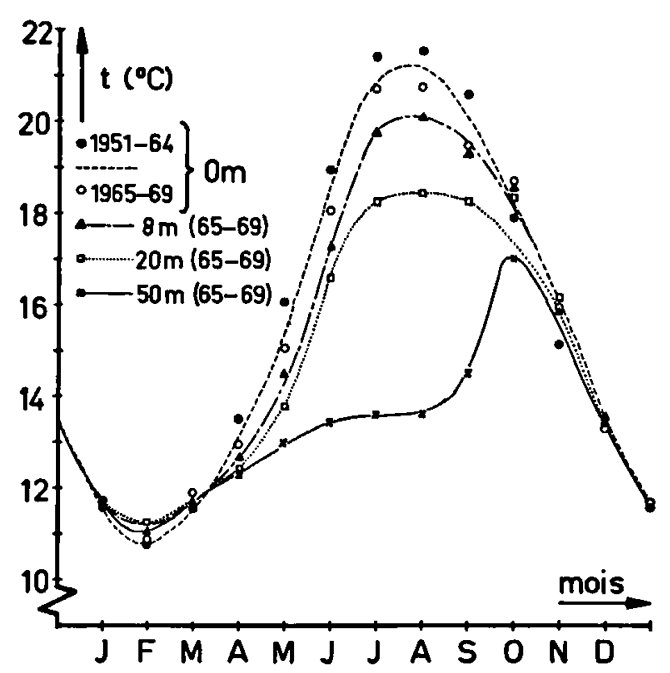

Fig. 7. Variations annuelles de la température à diverses profondeurs, dans la région de Banyuls-sur-Mer.

Comme il y a très peu de différences entre le cycle de température à $5 \mathrm{~m}$ et à $10 \mathrm{~m}$, nous avons pris la moyenne de ces deux courbes, que nous appelons la courbe de température à $8 \mathrm{~m}$ de profondeur. Nous avons donc quatre courbes-type: celles de $0 \mathrm{~m}$, de $8 \mathrm{~m}$, de $20 \mathrm{~m}$ et de $50 \mathrm{~m}$ de profondeur. Ces courbes représentent respectivement une moyenne de température annuelle de $16,0,15,5,14,9$ et $13,4^{\circ} \mathrm{C}$.

Il ressort de ce graphique l'existence d'une phase homogène (températures sensiblement égales depuis la surface jusqu'à $50 \mathrm{~m}$ de profondeur). Cette phase dure de la mi-octobre jusqu'au mois d'avril. Au mois de mai débute la phase hétérogène: un réchauffement en surface dû à l'insolation, mais qui n'est pas suivi en profondeur. Il s'établit une thermocline qui se situe à des profondeurs qui varient de 15 à $30 \mathrm{~m}$.

Vers la fin de l'été (septembre) les vents provoquent un fort brassage des eaux qui fait baisser la température au-dessus de la thermocline, tandis que les eaux plus profondes sont réchauffées. C'est ainsi que la phase homogène se rétablit en octobre.

\section{Les mesures}

Aucune de nos stations ne se trouvant à la surface, il nous suffisait de vérifier que chaque station appartenait à l'une des courbes-type suivantes: la courbe des $8 \mathrm{~m}$, caractéristique des profondeurs audessus de la thermocline; la courbe des $50 \mathrm{~m}$, caractéristique des profondeurs au-dessous de la thermocline; la courbe des $20 \mathrm{~m}$, caractéristique des profondeurs où se trouve la thermocline pendant une partie seulement des jours de la phase hétérogène.

Pour ce faire, nous avons, pendant chaque plongée, fait des mesures à l'aide d'un thermomètre à mercure gradué à $0,1^{\circ} \mathrm{C}$. Ceci a permis de classer les stations dans trois catégories de température: élevée, moyenne et basse pour les courbes-type de 8,20 et $50 \mathrm{~m}$ respectivement.

Cependant des différences avec les courbes-type ont été observées, surtout pendant l'été 1973, où dans plusieurs stations la température a atteint des valeurs nettement supérieures aux moyennes indiquées dans la fig. 7. Nous citons quelques exemples:

$\begin{array}{llll}25 / 8 / 1973 & \text { Port-Vendres } & -23 \mathrm{~m} & 21,4^{\circ} \mathrm{C} \\ \text { 26/8/1973 Jetée Port-Vendres } & -10 \mathrm{~m} & 24,7^{\circ} \mathrm{C} \\ \text { 30/8/1973 } & \text { Cap Béar } & -28 \mathrm{~m} & 22,7^{\circ} \mathrm{C}\end{array}$

Ce réchauffement est dû à deux causes distinctes. D'abord, toutes ces stations se situent sur le fond, et non en pleine eau. De ce fait, la radiation est absorbée par le substrat en libérant de l'énergie calorifique, qui vient s'ajouter aux effets de l'énergie solaire au sein des eaux. Ensuite, l'été 1973 a été marqué par une longue absence des vents prédominants (Tramontane et Vent du Sud) de la région de Banyuls-sur-Mer. De ce fait, le brassage périodique qui joue normalement un rôle régulateur, ne s'est pas produit. La température a dépassé, à $10 \mathrm{~m}$ de profondeur, la température 
maximum (depuis près de 20 ans!) de $24,4^{\circ} \mathrm{C}$ signalée, en surface, en 1952 (Thiriot, 1966). Nous insistons sur ce phénomène particulier parce qu'il a eu des conséquences intéressantes pour la faune, que nous aborderons au paragraphe IX.

\section{LES AUTRES FACTEURS}

L'établissement d'une population stable dépend d'un grand nombre de facteurs écologiques: en dehors des facteurs physico-chimiques, les facteurs biotiques (prédation, concurrence, etc.) jouent un rôle important.

Il n'a pas été possible de tenir compte de tous ces facteurs pendant le présent travail. C'est pourquoi nous nous sommes concentré surtout sur les trois facteurs physiques présentés dans les paragraphes précédents, et qui nous semblaient être les facteurs les plus importants. Cependant, lors de nos plongées, nous avons relevé certaines autres données. La première, la composition faunistique des stations, sera traîtée dans le paragraphe suivant. C'est elle que nous mettrons en corrélation avec les conditions particulières des diverses stations, mais c'est elle aussi qui pourrait rendre compte de certains phénomènes d'ordre biotique.

Les autres facteurs, abiotiques, que nous avons notés sont:

- La profondeur de chaque station, mesurée à l'aide d'un profondimètre de plongée. Nos stations se trouvant toutes entre 0 et $40 \mathrm{~m}$ de profondeur, nous avons voulu vérifier (a posteriori) si nous n'avions pas eu des préférences pour certaines zones. Nous avons divisé la profondeur en 4 classes: de 0 à $9 \mathrm{~m}$, de 10 à $19 \mathrm{~m}$, de 20 à $29 \mathrm{~m}$ et de 30 à $39 \mathrm{~m}$. Dans ces classes on trouve respectivement $2,6,6$ et 5 stations. Il n'y a que la zone près de la surface (pauvre en Octocoralliaires) qui soit mal représentée, les autres zones ayant reçu à peu près la même attention.

- La nature du substrat dur (roche ou coralligène).

- La pente du substrat, que nous avons mesurée à l'aide d'un clinomètre, et que nous avons ensuite divisée en trois classes:

horizontale ou subhorizontale

en pente moyenne

verticale, surplombs et grottes $>80^{\circ}$.

- La présence ou l'absence de dépôt vaseux sur le substrat.

Une objection que l'on pourrait faire est que ces paramètres ne sont pas tous indépendants: en effet l'énergie lumineuse est corrélée à la profondeur et à la pente du substrat; le courant et la température sont également liés à la profondeur, et le coralligène est le type de substrat que l'on ne rencontre qu'à partir d'un certain seuil d'obscurité. C'est la tâche de l'analyse statistique de mettre en évidence la nature et l'importance de ces inter-dépendances (voir paragraphe VIII, 1).

Nous n'avons pas fait d'observations sur la composition chimique de l'eau de mer, parce que nous avons estimé que l'eau était relativement homogène, à tel point que sa composition chimique ne pourrait rendre compte de variations brusques dans la composition faunistique du fond.

\section{LES ANIMAUX}

Le but final de cette étude était de pouvoir définir les seuils de certains paramètres écologiques, entre lesquels chacune des espèces étudiées peut se développer de façon optimale. Pour cela nous avons voulu travailler avec des animaux qui devaient répondre à plusieurs critères:

- à des fins de comparabilité, ils devaient vivre tous soit sur substrat meuble, soit sur substrat dur,

- ils devaient exister en nombre important sur les fonds étudiés, ceci pour des besoins statistiques,

- ils devaient être facilement trouvables et reconnaissables sur le terrain par un plongeur,

- ils devaient appartenir à un même groupement taxonomique mais comporter plusieurs espèces différentes, ceci afin de permettre une comparaison.

Le choix s'est porté sur les Octocoralliaires du substrat dur, très fréquents dans la région de Banyuls-sur-Mer. Les espèces suivantes ont fait l'objet de notre étude:

Eunicella stricta (Bertoloni, 1810),

Lophogorgia sarmentosa (Esper, 1794),

Paramuricea clavata (Risso, 1826),

Parerythropodium coralloides (Pallas, 1766),

Alcyonium acaule (Marion, 1878),

Corallium rubrum (Linnaeus, 1758).

L'aspect externe des colonies de ces espèces est nettement différent, tant par la forme que par la coloration, de façon que le plongeur ne puisse les confondre.

A chaque station nous avons effectué des comptages à l'aide d'un cadre métallique de forme carrée et de $50 \mathrm{~cm}$ de côté. Nous avons compté 
toutes les colonies de taille supérieure à $2 \mathrm{~cm}$, c'est à dire des colonies agées de plus d'un an. Chaque station comportant une aire plus ou moins grande, mais relativement homogène quant à sa composition faunistique, nous avons effectué entre 10 et 20 comptages par station (entre $2,5 \mathrm{~m}^{2}$ et $5 \mathrm{~m}^{2}$ ). Nous avons ensuite calculé la présence moyenne de colonies de chaque espèce par mètre carré, arrondi à un nombre entier. Une exception a été faite pour les colonies de Parerythropodium coralloides. Ces animaux ont besoin d'axes dénudés de Gorgones pour substrat. Leur présence dépend donc du nombre de Gorgones disponibles. Nous avons exprimé la fréquence des colonies de Parerythropodium en nombre de colonies par nombre de colonies d'espèces susceptibles de servir d'hôte (Eunicella, Lophogorgia et Paramuricea).

Dans certaines stations, certaines espèces sont rencontrées de façon trop accidentelle pour qu'on en tienne compte lors de l'analyse statistique. Cependant nous reprendrons ces observations, qui sont souvent très significatives, dans le paragraphe IX.

\section{LES STATIONS}

Les stations sont portées sur la carte de la fig. 8. Les 9 stations figurant sur la carte sont souvent subdivisées en plusieurs stations sous-marines. Nous étions capable de retrouver les stations avec une grande précision (de l'ordre de $10 \mathrm{~m}$ ), grâce à des photographies prises de la côte à partir du bateau, une fois ce dernier ancré au-dessus d'une station pour la première fois.

\section{Description des stations. -}

\section{Station no. 1}

Nom: Jetée Port Vendres 1 (JPV1)

Profondeur: $7 \mathrm{~m}$

Substrat: roche

Pente: moyenne

Dépôt vaseux: non

Energie lumineuse: $1783 \mathrm{cal} / \mathrm{cm}^{2} / \mathrm{an}$

Courant: $140 \mathrm{~m} / \mathrm{h}$ ( 4 observations)

Température: moyenne annuelle $16,0^{\circ} \mathrm{C}$

Animaux: 27 Eunicella $/ \mathrm{m}^{2}$

\section{Station no. 2}

Nom: Jetée Port Vendres 2 (JPV2)

Profondeur: $16 \mathrm{~m}$

Substrat: roche

Pente: moyenne

Dépôt vaseux: non

Energie lumineuse: $845 \mathrm{cal} / \mathrm{cm}^{2} / \mathrm{an}$

Courant: $160 \mathrm{~m} / \mathrm{h}$ (4 observations)

Température: moyenne annuelle $15,5^{\circ} \mathrm{C}$
Animaux: 22 Eunicella/m²

$$
7 \text { Lophogorgia/m² }
$$

Station no. 3

Nom: Jetée Port Vendres 3 (JPV3)

Profondeur: $18 \mathrm{~m}$

Substrat: roche

Pente: verticale

Dépôt vaseux: non

Energie lumineuse: $698 \mathrm{cal} / \mathrm{cm}^{2} / \mathrm{an}$

Courant: $135 \mathrm{~m} / \mathrm{h}$ (4 observations)

Température: moyenne annuelle $14,9^{\circ} \mathrm{C}$

Animaux: 13 Eunicella/m²

$$
1 \text { Lophogorgia/m } \mathrm{m}^{2}
$$

Station no. 4

Nom: Port Vendres 1 (PV1)

Profondeur: $20 \mathrm{~m}$

Substrat: roche

Pente: (sub)horizontale

Dépôt vaseux: non

Energie lumineuse: $579 \mathrm{cal} / \mathrm{cm}^{2} / \mathrm{an}$

Courant: $135 \mathrm{~m} / \mathrm{h}$ (4 observations)

Température: moyenne annuelle $14,9^{\circ} \mathrm{C}$

Animaux: 34 Eunicella/m²

$$
\begin{aligned}
& 3 \text { Lophogorgia/m } \mathrm{m}^{2} \\
& 1 \text { Parerythropodium } / \mathrm{m}^{2}(0,027 \\
& \text { par Gorgone) }
\end{aligned}
$$

Station no. 5

Nom: Port Vendres 2 (PV2)

Profondeur: $22 \mathrm{~m}$

Substrat: coralligène

Pente: verticale

Dépôt vaseux: non

Energie lumineuse: $313 \mathrm{cal} / \mathrm{cm}^{2} / \mathrm{an}$

Courant: $85 \mathrm{~m} / \mathrm{h}$ (4 observations)

Température: moyenne annuelle $14,9^{\circ} \mathrm{C}$

Animaux: 2 Eunicella $/ \mathrm{m}^{2}$

2 Parerythropodium/ $/ \mathrm{m}^{2}$ (0,66 par Gorgone)

1 Lophogorgia/m²

15 Alcyonium $/ \mathrm{m}^{2}$

Station no. 6

Nom: Port Vendres 3 (PV3)

Profondeur: $24 \mathrm{~m}$

Substrat: roche (morceaux de schiste)

Pente: horizontale

Dépôt vaseux: oui

Energie lumineuse: $332 \mathrm{cal} / \mathrm{cm}^{2} / \mathrm{an}$

Courant: $85 \mathrm{~m} / \mathrm{h}$ ( 4 observations)

Température: moyenne annuelle $14,9^{\circ} \mathrm{C}$

Animaux: 20 Eunicella/m²

$$
\begin{aligned}
& 12 \text { Lophogorgia/m } \\
& 1 \text { Parerythropodium/m² } \\
& \text { (0,031 par Gorgone) }
\end{aligned}
$$

Station no. 7

Nom: Cap Béar 1 (BEA1)

Profondeur: $27 \mathrm{~m}$

Substrat: roche

Pente: (sub)horizontale

Dépôt vaseux: oui

Energie lumineuse: $305 \mathrm{cal} / \mathrm{cm}^{2} / \mathrm{an}$ 
Courant: $390 \mathrm{~m} / \mathrm{h}$ (5 observations)

Température: moyenne annuelle $14,9^{\circ} \mathrm{C}$

Animaux: 49 Eunicella/m ${ }^{2}$

$$
\begin{aligned}
& 11 \text { Lophogorgia/m } \mathrm{m}^{2} \\
& 8 \text { Parerythropodium } / \mathrm{m}^{2} \\
& (0,133 \text { par Gorgone) } \\
& 13 \text { Alcyonium } / \mathrm{m}^{2}
\end{aligned}
$$

Station no. 8

Nom: Cap Béar 2 (BEA2)

Profondeur: $22 \mathrm{~m}$

Substrat: roche

Pente: (sub)horizontale

Dépôt vaseux: non

Energie lumineuse: $481 \mathrm{cal} / \mathrm{cm}^{2} / \mathrm{an}$

Courant: $240 \mathrm{~m} / \mathrm{h}$ ( 4 observations)

Température: moyenne annuelle $14,9^{\circ} \mathrm{C}$

Animaux: 42 Eunicella/m $\mathrm{m}^{2}$

$$
\begin{aligned}
& 2 \text { Lophogoria/m } \mathrm{m}^{2} \\
& 5 \text { Parerythropodium } / \mathrm{m}^{2} \\
& \text { (0,114 par Gorgone) } \\
& 1 \text { Alcyonium } / \mathrm{m}^{2}
\end{aligned}
$$

Station no. 9

Nom: Cap de l'Abeille 1 (ABE1)

Profondeur: $36 \mathrm{~m}$

Substrat: coralligène

Pente: (sub)horizontale

Dépôt vaseux: oui

Energie lumineuse: $139 \mathrm{cal} / \mathrm{cm}^{2} / \mathrm{an}$

Courant: $180 \mathrm{~m} / \mathrm{h}$ (3 observations)

Température: moyenne annuelle $13,4^{\circ} \mathrm{C}$

Animaux: 3 Eunicella/m²

$$
\begin{aligned}
& 1 \text { Parerythropodium } / \mathrm{m}^{2} \\
& \text { (0,33 par Gorgone) }
\end{aligned}
$$

Station no. 10

Nom: Cap de l'Abeille 2 (ABE2)

Profondeur: $36 \mathrm{~m}$

Substrat: coralligène

Pente: verticale

Dépôt vaseux: oui

Energie lumineuse: $67 \mathrm{cal} / \mathrm{cm}^{2} / \mathrm{an}$

Courant: $110 \mathrm{~m} / \mathrm{h}$ ( 3 observations)

Température: moyenne annuelle $13,4^{\circ} \mathrm{C}$

Animaux: 2 Eunicella/m $\mathrm{m}^{2}$

$$
\begin{aligned}
& 1 \text { Parerythropodium } / \mathrm{m}^{2} \\
& \text { (0,5 par Gorgone) }
\end{aligned}
$$

Station no. 11

Nom: Cap de 1'Abeille 3 (ABE3)

Profondeur: $36 \mathrm{~m}$

Substrat: coralligène

Pente: grottes, failles

Dépôt vaseux: oui

Energie lumineuse: $35 \mathrm{cal} / \mathrm{cm}^{2} / \mathrm{an}$

Courant: $7 \mathrm{~m} / \mathrm{h}$ ( 3 observations)

Température: moyenne annuelle $13,4^{\circ} \mathrm{C}$

Animaux: 25 Corallium $/ \mathrm{m}^{2}$ (approximativement)

Station no. 12

Nom: Rédéris 1A (RED1A)

Profondeur: $15 \mathrm{~m}$

Substrat: roche/coralligène

Pente: verticale
Dépôt vaseux: non

Energie lumineuse: $177 \mathrm{cal} / \mathrm{cm}^{2} / \mathrm{an}$

Courant: $115 \mathrm{~m} / \mathrm{h}$ ( 9 observations)

Température: moyenne annuelle $14,9^{\circ} \mathrm{C}$

Animaux: 46 Paramuricea $/ \mathrm{m}^{2}$

1 Parerythropodium $/ \mathrm{m}^{2}$

(0,022 par Gorgone)

Station no. 13

Nom: Rédéris 1B (RED1B)

Profondeur: $18 \mathrm{~m}$

Substrat: roche/coralligène

Pente: surplomb

Dépôt vaseux: non

Energie lumineuse: $49 \mathrm{cal} / \mathrm{cm}^{2} / \mathrm{an}$

Courant: $70 \mathrm{~m} / \mathrm{h}$ (8 observations)

Température: moyenne annuelle $14,9^{\circ} \mathrm{C}$

Animaux: 46 Paramuricea $/ \mathrm{m}^{2}$

1 Parerythropodium $/ \mathrm{m}^{2}$

(0,022 par Gorgone)

Station no. 14

Nom: Rédéris 1C (RED1C)

Profondeur: $23 \mathrm{~m}$

Substrat: roche/coralligène

Pente: surplomb

Dépôt vaseux: non

Energie lumineuse: $40 \mathrm{cal} / \mathrm{cm}^{2} / \mathrm{an}$

Courant: $72 \mathrm{~m} / \mathrm{h}$ (8 observations)

Température: moyenne annuelle $14,9^{\circ} \mathrm{C}$

Animaux: 46 Paramuricea $/ \mathrm{m}^{2}$ 1 Parerythropodium $/ \mathrm{m}^{2}$ (0,022 par Gorgone)

Station no. 15

Nom: Rédéris 2 (RED2)

Profondeur: $19 \mathrm{~m}$

Substrat: roche

Pente: (sub)horizontale

Dépôt vaseux: non

Energie lumineuse: $635 \mathrm{cal} / \mathrm{cm}^{2} / \mathrm{an}$

Courant: $230 \mathrm{~m} / \mathrm{h}$ ( 3 observations)

Température: moyenne annuelle $14,9^{\circ} \mathrm{C}$

Animaux: 47 Eunicella/m²

2 Parerythropodium $/ \mathrm{m}^{2}$

(0,043 par Gorgone)

Station no. 16

Nom: Rédéris 3A (RED3A)

Profondeur: $7 \mathrm{~m}$

Substrat: roche

Pente: moyenne

Dépôt vaseux: non

Energie lumineuse: $1078 \mathrm{cal} / \mathrm{cm}^{2} / \mathrm{an}$

Courant: $540 \mathrm{~m} / \mathrm{h}$ (3 observations)

Température: moyenne annuelle $15,5^{\circ} \mathrm{C}$

Animaux: 20 Eunicella/m² (approximativement)

Station no. 17

Nom: Rédéris 3B (RED3B)

Profondeur: $16 \mathrm{~m}$

Substrat: roche

Pente: moyenne

Dépôt vaseux: non

Energie lumineuse: $743 \mathrm{cal} / \mathrm{cm}^{2} / \mathrm{an}$ 


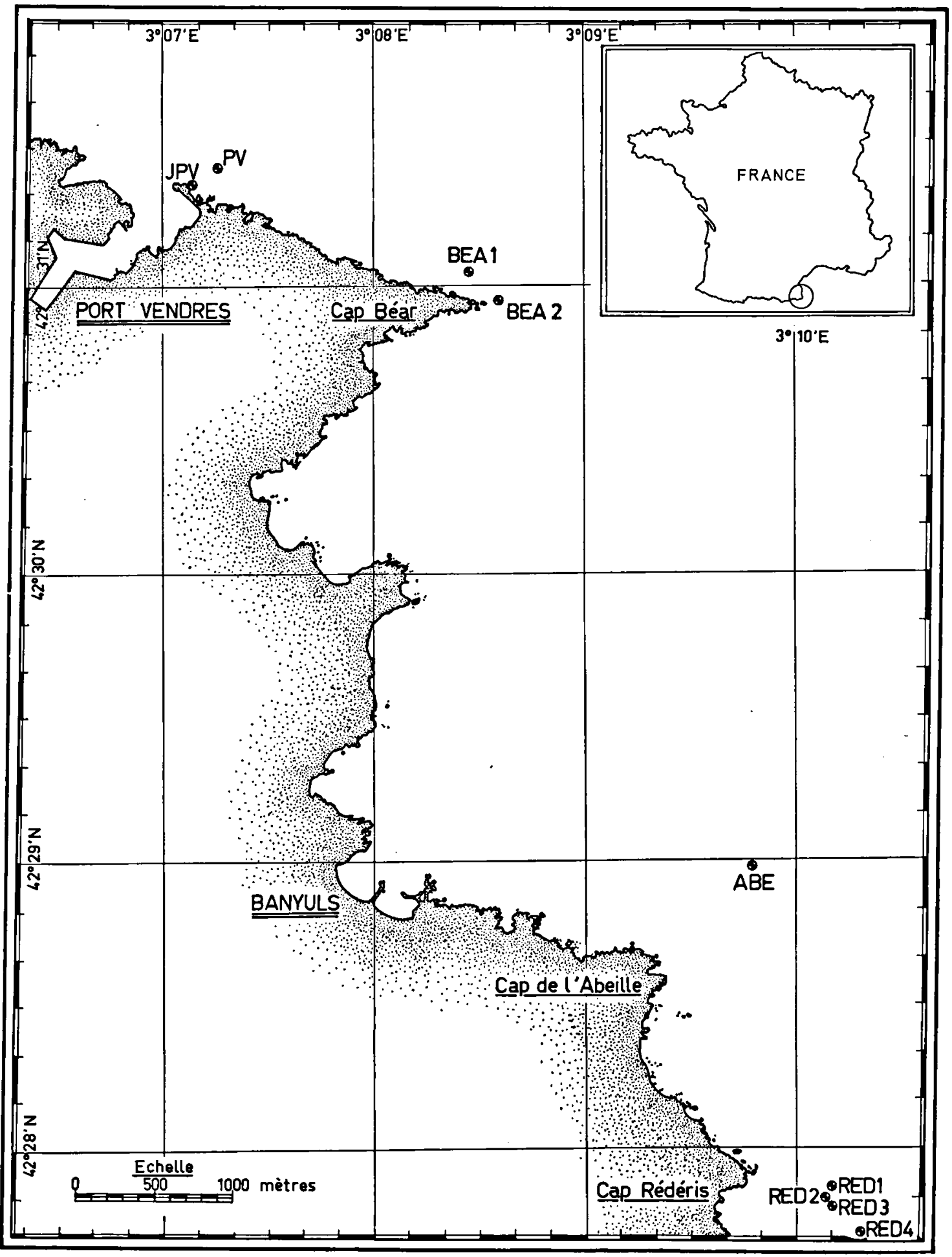

Fig. 8. Carte de la région prospectée, montrant l'emplacement des diverses stations. Pour la signification des abréviations utilisées, voir $\S$ VII. 
Courant: $75 \mathrm{~m} / \mathrm{h}$ ( 2 observations)

Température: moyenne annuelle $14,9^{\circ} \mathrm{C}$

Animaux: 30 Eunicella $/ \mathrm{m}^{2}$ (approximativement)

Station no. 18

Nom: Rédéris 4A (RED4A)

Profondeur: $36 \mathrm{~m}$

Substrat: coralligène

Pente: (sub)horizontale

Dépôt vaseux: oui

Energie lumineuse: $95 \mathrm{cal} / \mathrm{cm}^{2} / \mathrm{an}$

Courant: $55 \mathrm{~m} / \mathrm{h}$ ( 5 observations)

Température: moyenne annuelle $13,4^{\circ} \mathrm{C}$

Animaux: 10 Eunicella $/ \mathrm{m}^{2}$

4 Parerythropodium $/ \mathrm{m}^{2}$

( 0,4 par Gorgone)

8 Alcyonium $/ \mathrm{m}^{2}$

Station no. 19

Nom: Rédéris 4B (RED4B)

Profondeur: $36 \mathrm{~m}$

Substrat: coralligène

Pente: grottes, failles

Dépôt vaseux: oui

Energie lumineuse: $25 \mathrm{cal} / \mathrm{cm}^{2} / \mathrm{an}$

Courant: $0 \mathrm{~m} / \mathrm{h}$ ( 5 observations)

Température: moyenne annuelle $13,4^{\circ} \mathrm{C}$

Animaux: 15 Corallium $/ \mathrm{m}^{2}$ (approximativement)

\section{L'ANALYSE STATISTIQUE}

\section{L'analyse factorielle des correspondances}

Avant d'aborder les résultats de l'analyse statistique nous ferons un bref exposé sur les méthodes employées. Il existe des ouvrages consacrés aux méthodes de statistique modernes où le lecteur intéressé trouvera décrit avec plus de détail la théorie et la pratique de ces méthodes (par exemple: Lebart \& Fénelon, 1971).

L'analyse factorielle est employée dans le cas où nous avons un grand nombre d'observations portant sur un grand nombre de variables. Du point de vue statistique, chacune de nos stations représente une seule observation. Cette observation porte sur un certain nombre de variables, telles la température moyenne annuelle de la station ou l'énergie lumineuse reçue pendant une année, et à chaque station sont associées six caractères mesurés par le nombre de colonies de chaque espèce.

Nous avons donc $n$ observations portant sur $p$ variables. Nous pouvons les écrire dans un tableau rectangulaire à $n$ lignes et $p$ colonnes. Ceci donne la matrice $R$, de terme général $r_{i j}$, où $i$ est l'indiceligne et $j$ l'indice-colonne. Cette matrice s'écrirait ainsi:

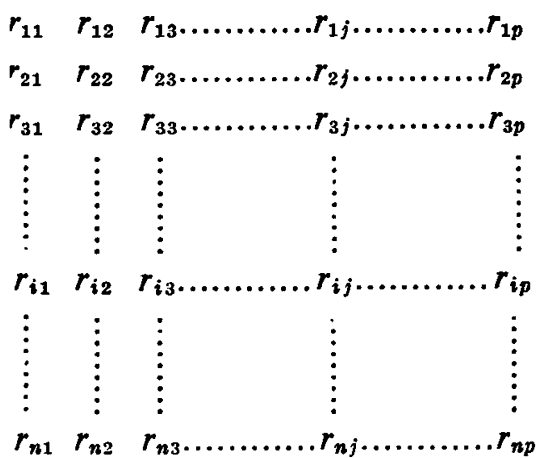

Appelons $r_{o j}$ le vecteur-colonne des $n$ observations de la variable $j$ et $r_{i v}$ celui des valeurs des $p$ variables pour la $i$-ème observation. Dans cette notation $o$ va de 1 à $n$ (pour chaque variable, il y a $n$ observations) et $v$ va de 1 à $p$ (chaque observation porte sur $p$ variables). Ce tableau de valeurs numériques peut donner lieu à deux représentations géométriques:

- dans l'espace $\mathbf{R}^{n}$ (espace à $n$ dimensions): un nuage de $p$ points, dont les coordonnées sont les composantes des vecteurs $r_{01}, r_{02}, \ldots . ., r_{o p}$

- dans l'espace $\mathbf{R}^{p}$ (espace à $p$ dimensions): un nuage de $n$ points, dont les coordonnées sont les composantes des vecteurs $r_{1 v}, r_{2 v}, \ldots \ldots, r_{n v}$

Plaçons-nous dans $\mathbf{R}^{p}$. Nous cherchons à décrire la forme du nuage de $n$ points et sa position par rapport à l'origine par un espace à deux dimensions. Autrement dit, nous cherchons le plan qui a la propriété de donner l'image la moins déformée du nuage, quand on projette tous les points du nuage sur lui. On dit que ce plan conserve le mieux l'inertie du nuage.

Il en résulte une représentation graphique où la proximité des points est significative de leur degré d'interdépendance, bien que l'on n'ait spécifié ni la nature ni l'importance de ces relations au départ. Quant à la signification de chacun des deux axes qui définissent le plan (les deux axes qui conservent le mieux l'inertie du nuage), elle n'est pas définie à l'avance, et doit être déduite de la position des $n$ points par rapport à ces axes.

En pratique, nous avons 19 stations (voir paragraphe précédent), chacune définie par sa composition faunistique; et 19 variables, parce que nous avons divisé chacune des variables d'origine en plusieurs modalités, chaque modalité devenant une nouvelle variable. Ces variables sont: 


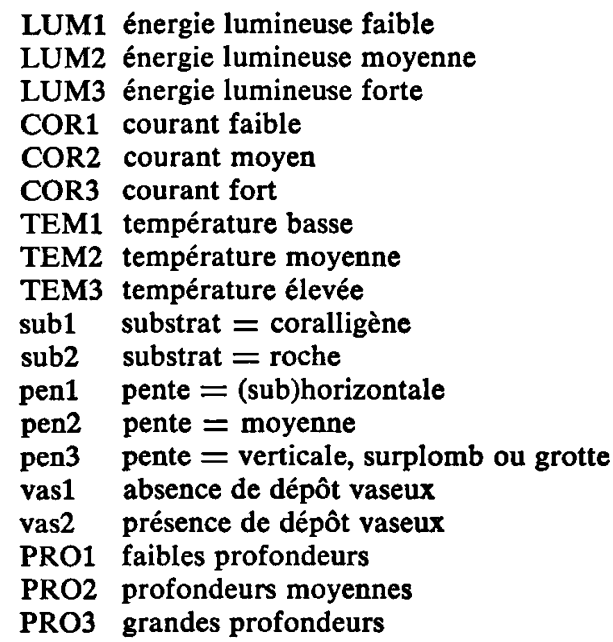

LUM1 énergie lumineuse faible

COR3 courant fort

TEM1 température basse

TEM2 température moyenne

sub1 substrat $=$ coralligène

sub2 substrat $=$ roche

pen1 pente $=$ (sub)horizontale

pen2 pente $=$ moyenne

pen 3 pente $=$ verticale, surplomb ou grotte

absence de dépôt vaseux

PRO1 faibles profondeurs

PRO3 grandes profondeurs

Sur le graphique de la fig. 9 tous ces points sont projetés, ainsi que les six espèces étudiées. On constate immédiatement sur ce graphique la nette corrélation entre la profondeur, la température et la luminosité. Cette conclusion, par sa trivialité, montre bien la robustesse de la méthode.

Avant d'aborder les autres conclusions que l'on pourrait tirer de ce graphique, essayons de trouver la signification des axes. Il ressort de l'analyse que les deux axes conservent près de $60 \%$ de l'inertie du nuage, ce qui est satisfaisant pour le nombre de variables (12) que nous avons ici $^{2}$ ). Cela veut dire que le graphique de la fig. 9 est assez fiable. Quelle est donc la signification de ces deux axes?

Pour l'axe horizontal, l'interprétation est assez simple: en allant de gauche à droite dans la figure, il semble que nous plongeons vers le fond: la luminosité diminue, l'eau devient de plus en plus froide et de plus en plus calme. Toutes ces conditions sont corrélées avec la profondeur. Nous pouvons donc appeler l'axe horizontal „axe de profondeur".

L'interprétation de l'axe vertical est plus difficile. Cependant, il nous semble qu'en allant au-dessus de l'axe de profondeur nous rencontrons les conditions "moyennes", tandis que les conditions ,extrêmes" se rencontrent dans le bas de la figure. La pente et le courant ne semblent pas vouloir s'en tenir à cette conclusion. Cependant il faut noter que pen2 n'est rencontré que dans 4 cas, et se comporte peutêtre, par sa rareté relative, comme une condition „extrême". On rencontre pen2 aux stations 1, 2 , 16 et 17 . Un coup d'oeil sur la figure montre qu'elle

2) Auparavant, nous avions défini 19 variables. Mais il y a en fait 7 liaisons linéaires. Par exemple: on a toujours LUM1 + LUM2 + LUM3 $=1$. Il reste donc 12 degrés de liberté.
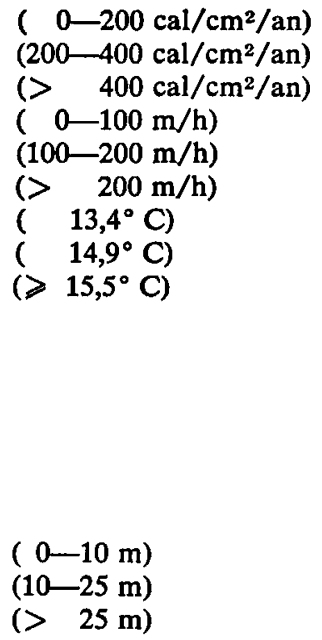

en rend très bien compte pour les trois premières stations. Pour le courant, l'explication est moins aisée, et nous ne nous aventurerons pas dans des théories à ce sujet.

Voyons maintenant ce que la figure nous apprend sur les animaux. Presque tous les animaux semblent préférer des conditions plutôt moyennes, sauf Corallium. Cette conclusion s'impose par la position des points par rapport aux deux axes. D'après l'analyse, l'ordre dans lequel on peut espérer rencontrer les espèces en partant de la surface est: Eunicella, Lophogorgia, Paramuricea, Parerythropodium, Alcyonium, Corallium. Ceci confirme nos observations en plongée. De même, les stations-type pour chacune des espèces seraient:

$\begin{array}{ll}\text { Eunicella stricta } & \text { JPV3, RED3B } \\ \text { Lophogorgia sarmentosa } & \text { RED1A } \\ \text { Paramuricea clavata } & \text { RED1B, RED1C } \\ \text { Parerythropodium coralloides } & \text { BEA1 } \\ \text { Alcyonium acaule } & \text { BEA1 } \\ \text { Corallium rubrum } & \text { ABE3, RED4B }\end{array}$

En effet, on rencontre les espèces dans les stations indiquées, sauf Lophogorgia sarmentosa, que l'on ne trouve pas à RED1A. Pour une interprétation plus détaillée nous renvoyons le lecteur au paragraphe IX.

\section{L'analyse de variances}

Il ressort de l'analyse factorielle que la distribution des espèces est liée à la profondeur. Malheureusement, les facteurs étudiés en détail (luminosité, courant et température) sont également fonction de la profondeur. Il est donc nécessaire de définir l'influence de chacun de ces facteurs pris à part sur la distribution de nos animaux.

Pour cela, nous nous sommes servi de l'analyse de variances. Chaque facteur écologique $\boldsymbol{A}$ peut être réparti en un certain nombre de modalités (les 


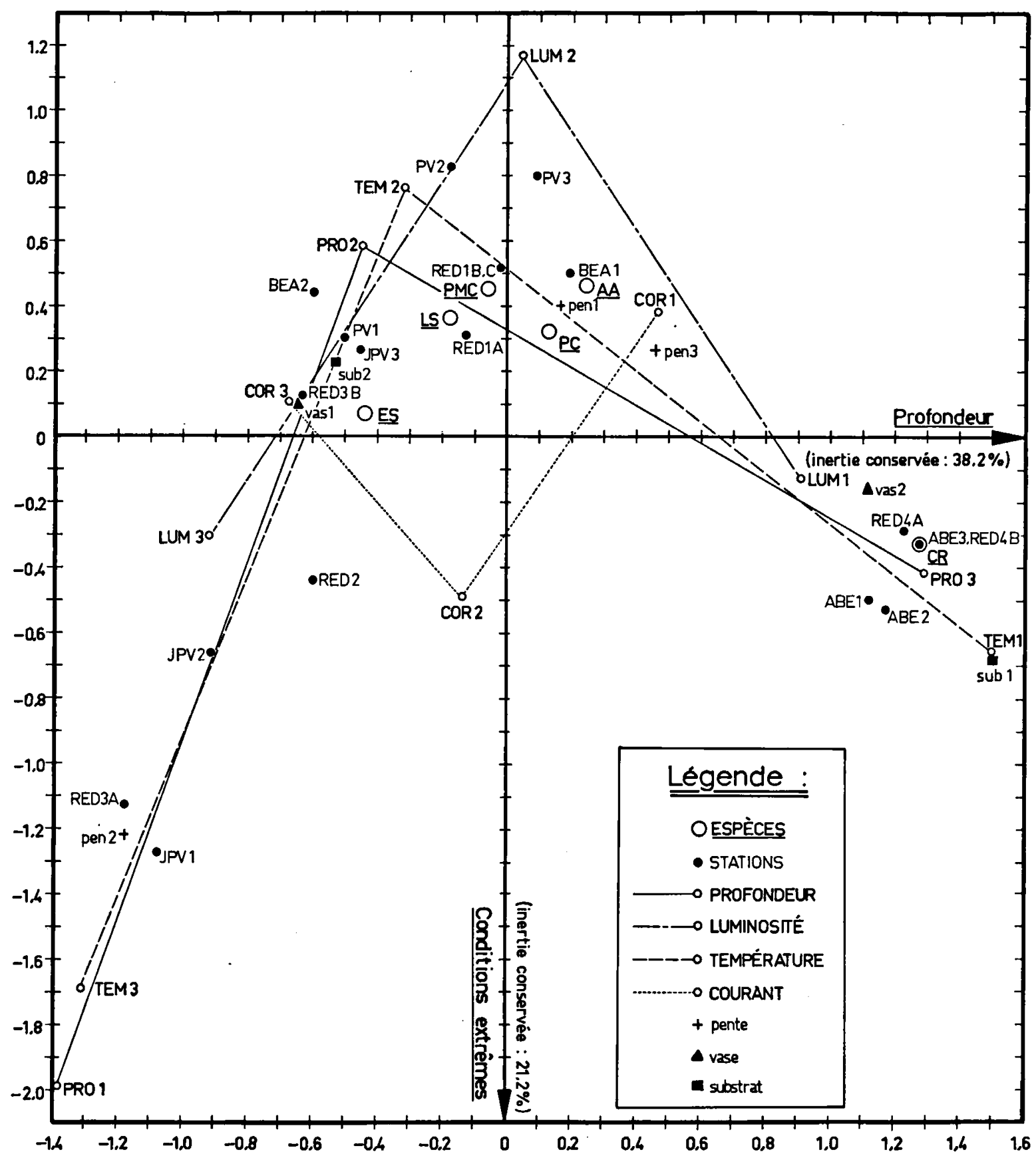

Fig. 9. Résultats de l'analyse factorielle des correspondances. Pour la signification des abréviations utilisées, cf. \$ VII pour les stations et $\S$ VIII, 1 pour les paramètres. Les espèces sont:

\section{ES Eunicella stricta \\ LS Lophogorgia sarmentosa \\ PMC Paramuricea clavata}

classes dans lesquelles nous allons diviser ce facteur) $A_{1}, A_{2}, \ldots \ldots, A_{k}$. Chaque modalité est un groupe expérimental. Le nombre d'observations au sein de chaque modalité est arbitraire: il ne doit pas être nécessairement le même.

Pour l'analyse, nous nous servirons des grandeurs

\section{PC Parerythropodium coralloides \\ AA Alcyonium acaule \\ CR Corallium rubrum}

suivantes (chaque fois $i=1,2, \ldots \ldots, k$ ):

$n_{i}=$ nombre d'observations pour la modalité $A_{i}$,

$n=\Sigma n_{i}=$ nombre total d'observations,

$A_{i}=$ somme des observations pour la modalité $A_{i}$, 
$X=\Sigma A_{i}=$ somme de toutes les observa, tions,

$Q_{i}=$ somme des carrés de toutes les observations pour la modalité $\boldsymbol{A}_{\boldsymbol{i}}$

$Q=\Sigma Q_{i}=$ somme des carrés de toutes les observations.

On peut, maintenant, formuler deux hypothèses:

- la moyenne des observations dans chaque modalité est la même

- les moyennes des observations dans les différentes modalités diffèrent entr'elles.

Partons de la première hypothèse, disant que toutes les observations appartiennent à la même distribution normale. A partir des observations on peut alors formuler trois estimateurs de la variance $\sigma^{2}$ de cette distribution:

a) Pour le total des observations la somme des carrés $q=Q-X^{2} / n$ est un estimateur de $(n-1) \sigma^{2}$

b) A l'intérieur de chaque modalité $A_{i}(i=1 \ldots k)$ la somme des carrés $q_{i}=Q_{i}-A_{i}{ }^{2} / n_{i}$ est un estimateur de $\left(n_{i}-1\right) \sigma^{2}$; en sommant pour les diverses modalités, $q_{0}=\Sigma q_{i}=Q-\Sigma A_{i}{ }^{2} / n_{i}$ est un estimateur de $(n-k) \sigma^{2}$ (variance intramodalités).

c) Il ressort des résultats précédents, par soustraction, que $q_{A}=q-q_{o}=\Sigma A_{i}{ }^{2} / n_{i}-X^{2} / n$ est un estimateur de $(k-1) \sigma^{2}$ (variance intermodalités).

Si l'hypothèse formulée est juste, $q /(n-1), q_{0}$ / $(n-k)$ et $q_{A} /(k-1)$ seront des estimateurs de la même variance $\sigma^{2}$. Si l'hypothèse est fausse, cependant, c'est à dire si la moyenne de la distribution varie d'une modalité à l'autre, $q_{0} /(n-k)$ restera un estimateur de $\sigma^{2}$, mais dans les deux autres grandeurs seront exprimées les différences entre les moyennes des différentes modalités, surtout dans la grandeur $q_{A} /(k-1)$. On peut donc vérifier l'hypothèse au moyen de la grandeur:

$$
F=\frac{q_{A} /(k-1)}{q_{\mathrm{o}} /(n-k)}
$$

qui a une distribution $F(k-1, n-k)$.

Dans notre cas, nous avons divisé chaque facteur en un grand nombre de classes, au moyen de bornes arbitraires. Ensuite, nous avons pris toutes les combinaisons possibles de deux bornes, définissant chaque fois trois modalités:

- la (les) classe(s) inférieure(s) à la lère borne;

- la (les) classe(s) comprise(s) entre les deux bornes;

- la (les) classe(s) supérieure(s) à la 2ème borne.

Pour chaque combinaison de deux bornes nous avons testé l'hypothèse selon laquelle la moyenne des colonies de l'espèce considérée par station est la même pour les trois modalités (hypothèse $H_{0}$ ). Avec $n-k=16$ (19 stations, 3 modalités)

$$
k-1=2 \text { ( } 3 \text { modalités) }
$$

on rejette l'hypothèse $H_{0}$ pour $F(16,2)$ :

$F>3,63$ avec $5 \%$ de chances de rejeter $H_{0}$ à tort;

$F>6,23$ avec $1 \%$ de chances de rejeter $H_{0}$ à tort;

$F>7,51$ avec $1 / 2 \%$ de chances de rejeter $H_{0}$ à tort.

Pour la luminosité nous avons pris les bornes

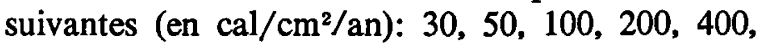
$600,800,1000$ et 1500 . Pour le courant (moyennes en $\mathrm{m} / \mathrm{h}$ ): $20,60,80,100,120,150,200,300$ et 500. Pour la température, enfin (moyennes annuelles en ${ }^{\circ} \mathrm{C}$ ): $14,0,15,2$ et 15,8 . Le calcul a donné les résultats suivants:

Pour la lumière (cal/ $\left.\mathrm{cm}^{2} / \mathrm{an}\right)$. -

Eunicella: au seuil de $1 / 2 \%$ on trouve les bornes suivantes: $30-200,50-200,100-200,100-$ $400,200-400,200-600,200-800,200-$ 1000 et $200-1500$, avec la plus grande valeur de $F(12,28)$ pour $200-800$.

Lophogorgia: au seuil de $1 / 2 \%$ une seule valeur de $F(9,74)$ est retenue, pour les bornes 200 400.

Paramuricea: aucune des valeurs de $F$ ne permet de rejeter $H_{0}$ avec une incertitude au seuil de $1 / 2 \%$ ou $1 \%$. Au seuil de $5 \%$ on trouve deux valeurs de $F(4,63$ et 3,79$)$ pour les bornes $30-$ 50 et $30-200$ respectivement.

Parerythropodium: au seuil de $1 \%$ une seule valeur de $F(7,35)$ est retenue pour les bornes $50-400$.

Alcyonium: au seuil de $1 / 2 \%$ une seule valeur de $F(8,33)$ est retenue pour les bornes $200-$ 400.

Corallium: au seuil de $1 \%$ une seule valeur de $F(6,70)$ est retenue pour les bornes $30-50$.

Pour le courant $(\mathrm{m} / \mathrm{h})$. -

Eunicella: au seuil de $1 / 2 \%$ on trouve les bornes suivantes: $20-200,60-200,80-200,100-$ $200,120-200,150-200,200-300$ et $200-500$, avec les plus grandes valeurs de $F(12,04$ et $12,21)$ pour $120-200$ et $200-500$.

Lophogorgia: on ne trouve qu'une seule valeur de $F(4,22)$ au seuil de $5 \%$ pour les bornes $300-500$.

Paramuricea: on ne trouve que deux valeurs de $F$ au seuil de $5 \%$, pour les bornes $60-80$ et $60-120$ respectivement. 
S. WEINBERG - ÉCOLOGIE DES OCTOCORALLIAIRES

Parerythropodium: aucune des valeurs de $F$ ne permet de tirer une conclusion de l'analyse pour ce facteur.

Alcyonium: une valeur de $F(4,09)$ au seuil de $5 \%$, pour les bornes $300-500$.

Corallium: le test à deux bornes donne un grand nombre de valeurs égales, parce que le Corallium est toujours trouvé en dehors des bornes choisies. Le test à une borne (voir plus loin) donnera un résultat plus compréhensible.

Pour la température $\left({ }^{\circ} \mathrm{C}\right)$. -

Il n'est pas permis de tirer une conclusion de l'analyse pour ce facteur: aucune des espèces ne donne lieu à des valeurs de $F$ supérieures à 3,63 .

Nous avons repris l'analyse des variances pour deux modalités. Parmi les mêmes bornes, nous n'en avons pris qu'une seule à la fois, et testé l'hypothèse que l'espèce est rencontrée aussi souvent à gauche qu'à droite de la borne. Les valeurs de $F$ sont: $F(17,1)=10,40(1 / 2 \%)$

$$
\begin{aligned}
& F(17,1)=8,40(1 \%) \\
& F(17,1)=4,45(5 \%)
\end{aligned}
$$

Cette analyse a donné les résultats suivants:

Pour la lumière (cal $\left./ \mathrm{cm}^{2} / \mathrm{an}\right)$. -

Eunicella: deux valeurs de $F(24,21$ et 11,60$)$ permettent de conclure au seuil de $1 / 2 \%$, pour les bornes 200 et 400 respectivement.

Lophogorgia: pas de conclusion possible.

Paramuricea: au seuil de 5\% deux bornes sont retenues: 50 et 200 respectivement.

Parerythropodium: pas de conclusion possible. Alcyonium: pas de conclusion possible.

Corallium: une valeur de $F(11,93)$ donne lieu à une conclusion au seuil de $1 / 2 \%$ pour la borne 50.

Pour le courant $(\mathrm{m} / \mathrm{h})$. -

Eunicella: au seuil de $1 / 2 \%$ on trouve deux valeurs de $F(13,26$ et 16,20$)$ pour les bornes 120 et 200 respectivement.

Lophogorgia: pas de conclusion possible.

Paramuricea: pas de conclusion possible.

Parerythropodium: pas de conclusion possible. Alcyonium: pas de conclusion possible.

Corallium: au seuil de $1 / 2 \%$ deux valeurs de $F$ : $243,37(!)$ et 24,11 pour les bornes 20 et 60 respectivement.

Pour la température $\left({ }^{\circ} \mathrm{C}\right)$. -

Eunicella: une seule valeur de $F(5,57)$ au seuil de $5 \%$, pour la borne 14,0 .

Lophogorgia: pas de conclusion possible.
Paramuricea: pas de conclusion possible.

Parerythropodium: pas de conclusion possible. Alcyonium: pas de conclusion possible.

Corallium: une valeur de $F(7,56)$ au seuil de $5 \%$, pour la borne 14,0 .

Les conclusions détaillées seront traitées dans le paragraphe suivant. Nous pouvons cependant conclure dès maintenant que l'analyse à 3 modalités donne plus de résultats que celle à 2 modalités. L'interprétation est simple: la présence de nos organismes dépend de 2 bornes pour chaque facteur. Une valeur supérieure au-dessus de laquelle l'animal ne trouve plus des conditions de vie optimales, et un seuil inférieur au-dessous duquel ces conditions ne sont pas remplies non plus.

\section{LES RÉSULTATS}

\section{Eunicella stricta}

C'est certainement l'espèce la plus tolérante, ce qui explique qu'on la trouve dans la plupart des stations. D'après l'analyse, elle se porterait bien dans des luminosités allant de 30 à $1500 \mathrm{cal} / \mathrm{cm}^{2} /$ an, avec une préférence pour les valeurs moyennes (200-800 cal $\left./ \mathrm{cm}^{2} / \mathrm{an}\right)$. Les valeurs extrêmes auxquelles nous l'avons rencontrée sont $67 \mathrm{cal}$ $\mathrm{cm}^{2} /$ an (ABE2) et $1783 \mathrm{cal} / \mathrm{cm}^{2} / \mathrm{an}$ (JPV1).

L'analyse donne également une fourchette de courants de 20 à $500 \mathrm{~m} / \mathrm{h}$ avec une préférence pour les courants moyens et forts $(>120 \mathrm{~m} / \mathrm{h})$. Valeurs extrêmes relevées: $55 \mathrm{~m} / \mathrm{h}$ (RED4A) et $540 \mathrm{~m} / \mathrm{h}$ (RED3A).

Elle n'a pas de préférence nette pour la température. On la rencontre surtout dans les endroits où la température moyenne annuelle est supérieure à $14,0^{\circ} \mathrm{C}$. Il faut noter cependant que dans tous les endroits où la température a dépassé $24^{\circ} \mathrm{C}$ pendant l'été 1973, les Eunicella sont morts jusqu'au niveau de la thermocline: ils n'ont pas supporté cette température élevée pendant plusieurs jours (observations à JPV1, JPV2, JPV3, RED3A, RED3B, au Cap Béar et à l'Ile Grosse, près du Laboratoire Arago).

Le manque d'oxygène accompagnant cette hausse de température doit être exclu comme cause pour deux raisons: d'abord les zooxanthelles symbiontes qui vivent dans les tissus de ces organismes produisent, aussi près de la surface, des quantités considérables d'oxygène (Brafield et al., 1965) dont peuvent profiter les polypes. Par ailleurs, dans l'une de ces stations (JPV2), parmi 
les Eunicella morts par suite du phénomène précité, nous avons trouvé des colonies vivantes de Lophogorgia, organismes semblables aux Eunicella pour leurs besoins en oxygène, dépourvus de zooxanthelles, qui ont parfaitement résisté à ces circonstances extrêmes!

\section{Lophogorgia sarmentosa}

Animal préférant des luminosités moyennes (200$400 \mathrm{cal} / \mathrm{cm}^{2} / \mathrm{an}$ ). Conditions extrêmes observées: $305 \mathrm{cal} / \mathrm{cm}^{2} / \mathrm{an}$ (BEA1) et $845 \mathrm{cal} / \mathrm{cm}^{2} / \mathrm{an}$ (JPV2).

Animal rencontré dans les zones de courant assez fort $(300-500 \mathrm{~m} / \mathrm{h})$, mais observé dans les conditions extrêmes de $85 \mathrm{~m} / \mathrm{h}$ (PV2, PV3) et $390 \mathrm{~m} / \mathrm{h}$ (BEA1).

L'espèce est indifférente à la température, voir paragraphe précédent.

La profondeur minimum à laquelle on rencontre l'espèce n'étant dictée ni par la turbulence, ni par la température, dépend donc de la luminosité.

L'analyse factorielle avait donné la station RED1A comme station-type. Les conditions de luminosité $\left(177 \mathrm{cal} / \mathrm{cm}^{2} / \mathrm{an}\right)$ et de courant (115 $\mathrm{m} / \mathrm{h}$ ) y semblent un peu faibles. Cependant, la raison majeure de l'absence de Lophogorgia doit être la concurrence par Paramuricea, espèce qui semble exclure toute autre Gorgone (même Eunicella) de son aire de distribution.

\section{Paramuricea clavata}

Espèce rencontrée en quantités considérables sous les surplombs de RED1, où elle est accompagnée des éponges calcaires Clathrina coriacea et $C$. primordialis, elle semble assez sciaphile. Les valeurs de luminosité $\left(30-200 \mathrm{cal} / \mathrm{cm}^{2} / \mathrm{an}\right)$ auxquelles on rencontre l'animal confirment cette observation. On s'attendrait à le rencontrer dans d'autres stations obscures. En effet, nous l'avons rencontré en faible nombre aux stations PV2 et ABE2 dans des conditions d'éclairement semblables.

L'animal est rencontré dans des conditions de turbulence faible à moyenne $(60-120 \mathrm{~m} / \mathrm{h})$.

L'animal semble indifférent à la température.

La luminosité régit sa présence, comme en témoigne sa répartition à RED1: nous avons effectué deux mesures de luminosité à $50 \mathrm{~cm}$ l'une de l'autre. Au premier endroit vivaient des Paramuricea, au deuxième des Eunicella. Or ces derniers recevaient 3,3 fois plus de lumière que les Paramuricea!

\section{Parerythropodium coralloides}

Comme nous l'avons déjà indiqué, le facteur écologique le plus important pour cette espèce est la présence d'autres Octocoralliaires susceptibles de servir de support. Nous avons rencontré Parerythropodium sur Eunicella, Lophogorgia et Paramuricea, de même que sur des coquilles d'Avicula hirundo, elles-mêmes fixées sur des Gorgones.

Cependant, Parerythropodium a une préférence pour des luminosités de $50-400 \mathrm{cal} / \mathrm{cm}^{2} / \mathrm{an}$, mais semble indifférent à la turbulence et la température.

Il existe des variétés différemment colorées de Parerythropodium (Weinberg, 1975). Bien que nous n'ayons pas étudié le rapport qui pouvait exister entre la distribution de ces variétés et les facteurs écologiques, nous n'avons remarqué les variétés blanches et roses que dans les stations profondes, tandis que la variété rouge est rencontrée à toutes les profondeurs où vit l'espèce.

\section{Alcyonium acaule}

Bien que l'espèce présente une très nette préférence pour des valeurs de luminosité allant de 200 à $400 \mathrm{cal} / \mathrm{cm}^{2} / \mathrm{an}$, nous l'avons rencontrée dans des conditions extrêmes de $95 \mathrm{cal} / \mathrm{cm}^{2} /$ an (RED4A) et $481 \mathrm{cal} / \mathrm{cm}^{2} / \mathrm{an}$ (BEA2).

De même, bien que l'espèce préfère des endroits turbulents $(300-500 \mathrm{~m} / \mathrm{h})$ nous l'avons trouvée à RED4A avec un courant moyen de $55 \mathrm{~m} / \mathrm{h}$.

La température n'a pas d'effet sur l'espèce.

Alcyonium doit être considéré comme un animal très tolérant, bien qu'il ait des préférences pour les endroits pas trop éclairés où le courant est assez fort.

\section{Corallium rubrum}

De tous les animaux que nous avons étudiés, c'est certainement l'espèce dont les préférences écologiques étaient les mieux connues. Ceci nous permettra de comparer nos résultats avec ceux publiés par d'autres auteurs (Laborel \& Vacelet, 1961; Laubier, 1966; Riedl, 1966; Barletta et al., 1968; Vighi, 1970; Jaubert, 1972).

Il ressort de ces études que l'animal est sciaphile, avec une préférence pour des valeurs de luminosité relative (par rapport à la surface) allant de $0,01 \%$ jusqu'à 0,07\% (Jaubert, 1972). Malheureusement, ce dernier auteur, bien qu'il donne la sensibilité spectrale relative de la cellule (sous-marine) de son 
appareil, ne définit pas la gamme de longueurs d'onde dans laquelle les valeurs de surface ont été mesurées.

En effet, avec notre appareil nous mesurons directement le rapport éclairement sous-marin/ éclairement en surface, dans la gamme de longueurs d'onde définies dans le paragraphe II,2. Pour les endroits à Corallium nous avons trouvé des valeurs allant de $0,1 \%$ jusqu'à $1 \%$. Ceci laisse à supposer que Jaubert (1972) a effectué les mesures en surface avec un appareil à thermopile, auquel cas il faudrait multiplier nos observations par $K=0,112$ (voir II,2) pour pouvoir comparer. Nous trouvons alors des valeurs relatives allant de $0,0112 \%$ à $0,112 \%$, valeurs qui correspondent bien avec celles que donne Jaubert.

De nos études, le caractère sciaphile de l'espèce ressort également, avec une préférence indiscutable pour des valeurs de luminosité inférieures à $50 \mathrm{cal} / \mathrm{cm}^{2} / \mathrm{an}$.

Il faut noter maintenant que nous avons rencontré des colonies de Corallium, en faible nombre, dans des endroits obscurs de PV2 et de RED1. Bien que les valeurs de luminosité pour ces endroits particuliers de la première station n'aient pas été mesurées, pour l'autre elles sont certainement inférieures à $50 \mathrm{cal} / \mathrm{cm}^{2} / \mathrm{an}$.

Ensuite, Corallium est un animal aimant les eaux calmes, avec un courant moyen inférieur à $60 \mathrm{~m} / \mathrm{h}$. Il faut noter cependant que dans les profondeurs où nous avons travaillé, l'espèce, par suite de sa sciaphilie, se comporte de façon cryptophile. Dans ces endroits, la turbulence est faible. En profondeur, où l'animal devient plus acrophile (Laborel \& Vacelet, 1961), il supporte probablement des courants plus forts. Parce que nous n'avons traité statistiquement que les endroits les plus profonds où l'on trouve Corallium, il ressort de l'analyse que l'espèce semble préférer des eaux froides. Mais les autres endroits où nous avons trouvé l'animal contredisent cette conclusion.

Pour le cas de Corallium, il faut citer un facteur biotique de grande importance. Le seul prédateur que l'espèce connaisse, l'homme plongeur, cause actuellement des ravages dans son habitat, surtout dans les petites grottes facilement accessibles des hauts-fonds de Rédéris qui sont pillées de façon systématique par les clubs de plongée de la région. Les stations plus profondes, telles les chenaux à Corallium de la station ABE ont reçues des visites désastreuses de la part de corailleurs professionnels. Il serait souhaitable que les Fédérations de Plongée et la Direction des Affaires Maritimes soient conscientes de leur responsabilité à ce sujet.

\section{CONCLUSION ET DISCUSSION}

D’aprés cette étude, il semble très possible, avec un calcul approprié, de définir les conditions écologiques dans le milieu sous-marin à partir de données relativement rares. Notre méthode de travail en scaphandre autonome s'est révélé satisfaisante.

Pour les espèces étudiées, la luminosité se détache nettement comme étant le facteur écologique le plus important. On pourrait cependant apporter une amélioration à ces observations en remplaçant les mesures relatives et le calcul approché (,,jour-type" du mois, $k$ „moyen" du mois, transmission par mer "moyenne" en fonction de l'élévation du soleil) qui l'accompagne par des mesures absolues et continues de la luminosité dans les stations étudiées. Ceci est techniquement possible (Jaubert, 1972). Nous avons également testé des „compteurs de lumière” mis au point par Monsieur J. de Boer de l'Université d'Amsterdam. Ces appareils, munis de photorésistances à sensibilité mal définie et de piles ordinaires, ne servaient qu'à tester le principe du compteur dans les conditions sous-marines. Equipés de cellules au Sélénium et de batteries $\mathrm{Cd}-\mathrm{Ni}$, on pourrait leur donner la linéarité requise et l'autonomie nécessaire pour un bon fonctionnement pendant des périodes de plus d'un mois. Un nettoiement périodique du collecteur s'imposerait, qui pourrait être accompagné par le renouvellement des batteries.

Il est intéressant de soulever la question: de quelle nature est l'action qu'a la lumière sur les animaux étudiés? Bien que l'on comprenne le rôle que peut jouer la lumière pour la distribution de l'espèce à zooxanthelles (Eunicella), l'action sur les autres Octocoralliaires (et beaucoup d'autres benthontes) reste assez mystérieuse. Le temps semble venu pour tirer ces phénomènes au clair à l'aide d'expériences physiologiques.

Le courant est également intéressant comme facteur, mais une mesure plus précise et plus continue que celle que nous avons effectuée s'impose. Des transducteurs tels ceux employés par Forstner \& Rützler (1970) semblent indiqués.

Une dernière question que nous aimerions soulever ici est celle du comportement de la larve planula (Théodor, 1967; Vighi, 1970). En effet, il est fort probable que la distribution des espèces étudiées ne dépend que des conditions que rencontre la planula au moment de sa fixation sur le substrat: si ces conditions sont favorables, la larve se fixe et finalement une colonie se développe. Dans le cas contraire, la larve périt. Si cela 
était le cas, seules les conditions règnant pendant les mois d'été (mai-juillet) joueraient un rôle important. Cela ne change pas grand-chose pour les facteurs lumière et courant, mais le facteur température pourrait gagner en importance, à cause de l'existence de la thermocline pendant ces mois.

\section{REMERCIEMENTS}

Je remercie Monsieur le Professeur J. H. Stock de l'Institut de Zoologie taxonomique de l'Université d'Amsterdam, sous la responsabilité duquel j'ai pu entreprendre cette recherche. Je le remercie particulièrement pour la grande liberté qu'il m'a laissée dans le choix de mon sujet et dans mon travail.

Mes séjours en France m'ont été rendus possibles grâce à des contributions financières accordées par le Présidium de l'Université d'Amsterdam.

Pendant les trois dernières périodes de recherche, j'ai bénéficié d'une autorisation de séjour dans le Laboratoire Arago de l'Université de Paris, à Banyuls-sur-Mer. Je tiens à remercier Monsieur le Professeur $P$. Drach, Directeur, et Monsieur J. Soyer, Sous-directeur, de leur hospitalité.

Je remercie également Monsieur M. Panouse, qui m'a souvent aidé, et qui m'a fourni des données précieuses, ainsi que Monsieur $H$. Boutière, à qui je dois les enregistrements de l'ensoleillement à Banyuls-sur-Mer.

Enfin, je veux remercier tous ceux du Laboratoire Arago qui m'ont aidé et qui sont devenus mes amis.

Un grand merci va à mon père, Monsieur Edgar Weinberg, qui s'est privé de son bon bateau, le "CIAO", pendant une année, afin que je puisse m'en servir pour mon travail. Il en a été l'excellent capitaine pendant deux périodes de recherche.

Je n'ai pas besoin de dire grand-chose à tous les amis plongeurs qui m'ont aidé de façon magnifique: les plongeurs ne se parlent pas, ils se font signe... Je mentionne ici leurs noms (dans l'ordre du nombre de plongées effectuées): Messieurs Jaap de Boer, Patrick Weening et Philippe Onda, Mesdemoiselles Joana Paiva de Sousa et Francisca Zijlstra.

Je tiens à remercier également Monsieur André Bonneau, Président du Club Subaquatique de la Côte Vermeille qui m'a fait bénéficier de sa profonde connaissance des fonds sous-marins de la région prospectée.

Je suis heureux de pouvoir mentionner ici le rôle important qu'a joué Monsieur Dominique Strauss-Kahn du C.N.R.S., c'est lui qui s'est chargé du traitement statistique.

Enfin, c'est Madame Hélène Strauss-Kahn qui a corrigé mon texte français: qu'elle trouve ici l'expression de ma profonde reconnaissance.

\section{REFERENCES BIBLIOGRAPHIQUES}

Atkins, W. R. G. \& H. H. Poole, 1930. The photochemical and photo-electric measurement of submarine illumination. J. mar. biol. Ass. U.K., (n.s.) 16 (2): $509-514$.

Barletta, G., R. Marchetti \& M. Vighi, 1968. Ricerche sul corallo rosso: IV - Ulteriori osservazioni sulla distribuzione del corallo rosso nel Tirreno. Rc. Ist. lomb. Sci. Lett., 102 (B): 119-144.

Bhaud, M., G. Jacques \& C. Razouls, 1967. Données météorologiques et hydrologiques de la région de Banyuls-sur-Mer. Année 1965-1966 (point côtier). Vie Milieu, 18 (1B): 137-151.

Brafield, A. E., G. Chapman \& J. Théodor, 1965. Consommation d'oxygène chez Eunicella stricta (Bertoloni) Gorgone à zooxanthelles symbiotiques. Vie Milieu, 16 (2A): 647-654.

Chapman, G. \& J. ThÉodor, 1969. L'influence de la lumière sur la consommation $\mathrm{d}^{\prime} \mathrm{O}_{2}$ chez Eunicella stricta (Gorgone à zooxanthelles symbiotiques) et chez Paramuricea clavata. Vie Milieu, 20 (3A): 483-490.

Chardy, P., 1970. Ecologie des Crustacés Péracarides des fonds rocheux de Banyuls-sur-Mer. Amphipodes, Isopodes, Tanaidacés, Cumacés, infra et circalittoraux. Vie Milieu, 21 (3B): 657-727.

FORSTNER, H. \& K. RüTZLER, 1970. Measurements of the micro-climate in littoral marine habitats. Oceanogr. mar. Biol., 8: 225-249.

HolmES, R., 1957. Solar radiation, submarine daylight and photosynthesis. In: J. W. HeDGPETH ed. Treatise on marine ecology and paleoecology. Vol. I. Ecology. Mem. geol. Soc. Am., 67: 109-128.

JaCQues, G., C. RAzouls \& A. Thiriot, 1968. Données météorologiques et hydrologiques de la région de
Banyuls-sur-Mer année 1966-1967. Vie Milieu, 19 (1B): $185-195$.

-, — \& -, 1969a. Id., année 1967-1968. Vie Milieu, 20 (1B): $63-73$.

,$--\&-, 1969$ b. Climat et hydrologie à Banyuls-surMer (Golfe du Lion) 1965-1968. Vie Milieu, 20 (2B): 279-316.

,$--\&-, 1971$. Données météorologiques et hydrologiques de la région de Banyuls-sur-Mer (point côtier) année 1968-1969. Vie Milieu, 22 (1B): 61-74.

JAUBERT, J., 1972. Benthos: étude et mesure de l'éclairement. Etude et mesure d'un facteur écologique: l'éclairement. Réalisation d'un appareil enregistreur. Téthys, 3 (2): 205-246.

JERLOV, N. G., 1968. Optical oceanography: 1-194 (Elsevier, Amsterdam).

LABOREL, J. \& J. VACELET, 1961. Répartition bionomique du Corallium rubrum Lmck. dans les grottes et falaises sous-marines. Rapp. P.-v. Réun. Commn int. Explor. scient. Mer Méditerr., 16 (2): 465-469.

Landais, J., 1955. Mesure du coefficient d'extinction de l'eau de la Méditerranée dans la région de Banyuls. Vie Milieu. 6 (2): 210-224.

LAubier, L., 1966. Le coralligène des Albères. Monographie biocénotique. Annls Inst. océanogr., Paris, 43 (2): $139-316$.

LebART, L. \& J. P. FÉnElon, 1971. Statistique et informatique appliquées: i-v, 1-426 (Dunod, Paris).

RiedL, R., 1966. Biologie der Meereshöhlen: 1-636 (P. Parey, Hamburg/Berlin).

Roos, P. J., 1967. Growth and occurrence of the reef coral Porites astreoides Lamarck in relation to submarine radiance distribution: 1-72 (Elinkwijk, 
Utrecht) (Thèse Université d'Amsterdam).

SCHUMACHER, H., 1973. Morphologische und ökologische Anpassungen von Acabaria-Arten (Octocorallia) im Roten Meer an verschiedene Formen der Wasserbewegung. Helgoländer wiss. Meeresunters., 25 (4): 461472.

ThEODOR, J., 1963. Contribution à l'étude des Gorgones. III. Trois formes adaptatives d'Eunicella stricta en fonction de la turbulence et du courant. Vie Milieu, 14 (4): 815-818.

-, 1967. Contribution à l'étude des Gorgones (VII): Ecologie et comportement de la planula. Vie Milieu, 18 (2A): 291-301.

ThEODOR, J. \& M. Denizot, 1965. Contribution à l'étude des Gorgones (I): A propos de l'orientation d'organismes marins fixés végétaux et animaux en fonction du courant. Vie Milieu, 16 (1B): 237-241.
ThiRıot, A., 1966. Variations annuelles de la température de l'eau côtière superficielle de Banyuls-sur-Mer. Vie Milieu, 17 (1B): 243-252.

Velimirov, B., 1973. Orientation in the sea fan Eunicella cavolinii related to water movement. Helgoländer wiss. Meeresunters., 24 (1-4): 163-173.

VIGHI, M., 1970. Ricerche sul ciclo riproduttivo del corallo rosso (Corallium rubrum (L.)) del promontorio di Portofino. Atti Accad. naz. Lincei Memorie, (VIII) 10 (IIIa, 1): 1-26.

WeinberG, S., 1974. A relative irradiance meter for submarine ecological measurements. Neth. J. Sea Res., 8 (4): $354-360$.

- , 1975. Contribution à la connaissance de Parerythropodium coralloides (Pallas, 1766) Octocorallia, Alcyonacea. Beaufortia, 23 (298): 53-73.

Reçu: le 26 août 1974 